Algebraic $8 \mathcal{G}$ Geometric $\mathcal{T}_{\text {opology }}$

Volume 4 (2004) 439-472

Published: 27 June 2004

ATG

\title{
Embeddings of graph braid and surface groups in right-angled Artin groups and braid groups
}

\author{
JOHN CRISP \\ BERT Wiest
}

\begin{abstract}
We prove by explicit construction that graph braid groups and most surface groups can be embedded in a natural way in right-angled Artin groups, and we point out some consequences of these embedding results. We also show that every right-angled Artin group can be embedded in a pure surface braid group. On the other hand, by generalising to rightangled Artin groups a result of Lyndon for free groups, we show that the Euler characteristic -1 surface group (given by the relation $x^{2} y^{2}=z^{2}$ ) never embeds in a right-angled Artin group.
\end{abstract}

AMS Classification 20F36, 05C25; 05C25

Keywords Cubed complex, graph braid group, graph group, right-angled Artin group, configuration space

\section{Introduction and statement of results}

Right-angled Artin groups (which also go by the names of graph groups or partially commutative groups) are by definition those finitely presented groups whose defining relations consist simply of a certain number of commutation relations $x y=y x$ between elements $x, y$ of the generating set. It is customary and convenient to specify a right-angled Artin group by drawing a graph whose vertices correspond to the generators, and whose edges connect "commuting" vertices. These groups interpolate between the finite rank free and free abelian groups and share a number of important properties. They are linear (so residually finite) [16, 15, torsion free, and even bi-orderable as well as residually nilpotent [9]. Moreover, right-angled Artin groups act freely properly co-compactly on certain naturally associated CAT(0) cubed complexes, which implies that they are biautomatic (by [21, see also [26, 14]) and have quadratic isoperimetric inequality. Even better, these groups admit a polynomial time solution to the conjugacy problem [25, 27. Some but not all of the above properties are inherited by subgroups. On the other hand, subgroups of right-angled 
Artin groups can exhibit some surprising properties, depending on the structure of the underlying defining graph. Bestvina and Brady [4] were able, for instance, to distinguish for the first time between a variety of subtly different finiteness properties by looking at certain subgroups of these groups. Graph groups and their subgroups are therefore of considerable interest, not only for their attractive properties, but also as a varied source of examples.

In Section 2 we shall describe briefly the geometric properties of cubed complexes. We shall restate Gromov's combinatorial characterisation of the CAT(0) property for these complexes and shall prove a result which allows one to identify locally isometric embeddings between such spaces. This gives a method for constructing (quasi-convex) embeddings between groups which are fundamental groups of locally $\mathrm{CAT}(0)$ cubed complexes.

There are two classes of groups that we shall embed in right-angled Artin groups, using this technique: graph braid groups (i.e. fundamental groups of configuration spaces of $k$ disjoint points in a graph) which are treated in Section 3, and surface groups, treated in Section 4 All of these examples with the exception of exactly three closed surface groups can be successfully embedded.

When discussing graph braid groups it is more convenient to consider the larger class of "reduced graph braid groups" (defined in Section [3). These are the fundamental groups of certain cubed complexes which, unlike the usual configuration spaces, depend on the simplicial structure of the underlying graph. However, the braid group $B_{n}(G)$ over a graph $G$ is always isomorphic to the reduced graph braid group $R B_{n}\left(G^{\prime}\right)$ for a sufficiently fine subdivision $G^{\prime}$ of $G$ (see [1, 2, 3]). It was known already from [1, 3] that reduced graph braid groups act freely co-compactly on CAT(0) cubed complexes, and so are biautomatic, by the result of [21. In Section 3 we present a very natural embedding of each graph braid group in a right-angled Artin group which is induced by a locally isometric embedding of locally CAT(0) cubed complexes. As corollaries we have that graph braid groups are linear, bi-orderable, residually finite, residually nilpotent. In a future article we plan to give a proof that these groups also admit a polynomial time solution to the conjugacy problem.

There are two particular examples of (reduced) graph braid groups that are isomorphic to surface groups. These are the 2 -string braid groups $B_{2}\left(K_{3,3}\right) \cong$ $R B_{2}\left(K_{3,3}\right)$ and $B_{2}\left(K_{5}\right) \cong R B_{2}\left(K_{5}\right)$, where $K_{3,3}$ denotes the utilities graph, and $K_{5}$ the complete graph on 5 vertices (the smallest two non-planar graphs). The associated cubed complexes are squarings of non-orientable surfaces of Euler characteristic -3 and -5 respectively. See 1 for a discussion of these examples. Our construction embeds $B_{2}\left(K_{5}\right)$ into the right-angled Artin group 
whose defining graph is the 1-skeleton of the twofold quotient of the dodecahedron by the antipodal map. The group $B_{2}\left(K_{3,3}\right)$ embeds in the right-angled Artin group with defining graph $\Delta\left(K_{3,3}\right)$ shown in Figure 1 .

Results of [8] have shown that 'most' right-angled Artin groups contain nonabelian closed surface subgroups. More precisely, if the defining graph contains an induced (full) subgraph isomorphic to an $n$-cycle for $n \geq 5$, then the rightangled Artin group contains a non-abelian closed surface subgroup. However all the examples from [8] are orientable surface groups of relatively high genus (at least 5), which raises the question of exactly which surface groups can be embedded in a right-angled Artin group. This question is answered in Section 4 where we show that all surface groups (orientable, nonorientable, closed or with boundary) can be embedded in some right-angled Artin group, with three exceptions: the fundamental groups of the projective plane (which has 2-torsion), the Klein bottle (which has generalised torsion, so is not bi-orderable), and the nonorientable surface $S_{-1}$ with Euler characteristic -1 . We remark that the dual question of classifying those right-angled Artin groups (or Artin groups) which contain non-abelian (hyperbolic) closed surface subgroups remains open. Some partial results, notably in the case of finite and affine type Artin groups, are given in [11].

The group $\pi_{1}\left(S_{-1}\right)$, the last of the three exceptional surface groups mentioned above, has presentation $\left\langle x, y, z \mid x^{2} y^{2}=z^{2}\right\rangle$. In order to prove that this group cannot embed in any right-angled Artin group we give a topological proof that if elements $x, y, z$ of a right-angled Artin group satisfy the relation $x^{2} y^{2}=z^{2}$ then $x, y$ and $z$ mutually commute (see Theorem [7). This generalizes a classic result of Lyndon on free groups [18 to the case of right-angled Artin groups. Lyndon's result was shortly afterwards generalized to the following statement [20]: in a free group $F$, the relation $x^{m} y^{n}=z^{k}$ implies that $x, y$ and $z$ commute. We do not know whether this statement also holds true over an arbitrary right-angled Artin group.

In Section [6] we use somewhat different techniques to those above, to prove a result about embeddings in braid groups. We show that every right-angled Artin group (of rank $n$ ) may be embedded as a subgroup of the pure braid group (on $n$ strings) over some compact surface with boundary. Often this surface can be chosen to be planar, and so the right-angled Artin group can be embedded in a classical pure braid group $B_{l}$ for some $l \in \mathbb{N}$. This is discussed in Section 7 However, we are not able to answer the following question: does every right-angled Artin group embed in a classical braid group? We also leave open the question whether the surface group $\left\langle x, y, z \mid x^{2} y^{2}=z^{2}\right\rangle$ embeds in a braid group $B_{l}$ for some $l \in \mathbb{N}$. 
Acknowledgement We wish to thank Aaron Abrams for stimulating discussions in the early stages of this work. We are also grateful to Mark Sapir and Ilya Kapovich for helpful remarks on an earlier version of this manuscript, and to Luis Paris for bringing to our attention the work of Duchamp and Thibon on orderability and related properties of right-angled Artin groups. Part of the research was done while Bert Wiest was a postdoc at the Pacific Institute for the Mathematical Sciences (PIMS).

\section{Background on $\mathrm{CAT}(0)$ cube complexes}

By a cubed complex $X$ we shall mean a polyhedral complex in which each cell is a finite dimensional unit Euclidean cube. We refer to [5], pp.114-115, for a precise definition of polyhedral complex. Briefly, a cubed complex $X$ is an identification space defined by an equivalence relation on the disjoint union of a collection of Euclidean cubes, wherein the interior of each face of each cube is mapped injectively to $X$, and if two points $x, x^{\prime}$ lying in the interior of faces $F, F^{\prime}$ are identified then there is an isometry $h: F \rightarrow F^{\prime}$ such that $y$ and $h(y)$ are identified for all $y \in F$. For example, the $n$-torus obtained by identifying opposite faces of an $n$-cube is a cubed complex.

Every finite dimensional cubed complex $X$ is equipped with its so-called intrinsic metric $d$, defined by setting $d(x, y)$ to be the infimum of lengths of rectifiable paths from $x$ to $y$ in $X$ (where a rectifiable path is a path obtained by concatenating the images of finitely many straight line segments in cubes and the length of such a path is the sum of the Euclidean lengths of its segments). This actually defines a complete length metric on $X$ (see [5]).

By a $C A T(0)$ space we mean a geodesic metric space which satisfies the $\operatorname{CAT}(0)$ triangle inequality as defined, for example, in [13, 5]. This is a global nonpositive curvature condition which states that distances between points on a geodesic triangle are no greater than the distances between the corresponding points on a reference triangle of the same sidelengths in the Eulidean plane. By a locally $C A T(0)$ space we mean metric space in which every point has an open ball neighbourhood which is a CAT(0) space with the induced metric. The CartanHadamard Theorem [13, [5] states that if $X$ is a complete locally CAT(0) space then its universal cover $\widetilde{X}$ is a complete $\operatorname{CAT}(0)$ space.

By a local isometry between metric spaces we mean a map $f: X \rightarrow Y$ such that every point $p$ in $X$ has an open ball neighbourhood $N_{p}$ in $X$ such the restriction of $f$ to $N_{p}$ is an isometry with respect to the induced metric on $N_{p}$. 
If $X$ is a geodesic metric space, $Y$ a locally CAT(0) space, and $f: X \rightarrow Y$ a local isometry, then $X$ is also locally $\operatorname{CAT}(0)$ and $f$ is $\pi_{1}$-injective. In fact $f$ lifts to an isometric embedding of $\tilde{X}$ in $\widetilde{Y}$ (see [5], p.201).

Any combinatorial map between cubed complexes which takes each cube to a cube of the same dimension while respecting the face relation determines uniquely a map between the metric cubed complexes which is a local isometry on the interior of each cube. We call such a map a cubical map. The following Theorem will be useful in proving the existence of $\pi_{1}$-injective maps between cubed complexes. By a flag complex we mean a simplicial complex $K$ in which every set of $n+1$ vertices which spans a complete graph in the 1-skeleton of $K$ spans an $n$-simplex in $K$.

Part (1) of the following Theorem is due to Gromov [13, and is widely known. Part (2) seems to be the natural extension of Gromov's idea, and is essentially a consequence of Lemmas 1.4-1.6 of [6].

Theorem 1 (1) A finite dimensional cubed complex $Y$ is locally CAT(0) if and only the link of every vertex is a flag complex (or equivalently, if and only if, for every cube $C$ in $Y$, every triangle in the 1-skeleton of $L k(C, Y)$ bounds a 2-simplex).

(2) Let $X$ and $Y$ be finite dimensional cubed complexes and $\Phi: X \rightarrow Y$ a cubical map. Suppose that $Y$ is locally $C A T(0)$. Then the map $\Phi$ is a local isometry if and only if, for every vertex $x \in X$, the simplicial map $L k(x, X) \rightarrow L k(\Phi(x), Y)$ induced by $\Phi$ is injective with image a full subcomplex of $\operatorname{Lk}(\Phi(x), Y)$.

Moreover, in this case, $X$ is locally $C A T(0)$ and $\Phi$ is $\pi_{1}$-injective and lifts to an isometric embedding of $\widetilde{X}$ in $\tilde{Y}$.

Remark If, in part (2) of the Theorem, it is already known that the links in $X$ are flag (equivalently that $X$ is locally CAT(0)) then it is enough to check that the 1-skeleton of $L k(x, X)$ is mapped isomorphically onto a full subgraph of the 1 -skeleton of $L k(\Phi(x), Y)$, for all vertices $x \in X$, in order to show that $\Phi$ is a local isometry.

Proof We refer to [13] (see also [5]) for the proof of (1). Note that the link of any vertex in a cube complex is an all-right spherical complex, a piecewise spherical simplicial complex in which all edge lengths and all dihedral angles measure $\frac{\pi}{2}$. Note also that, in an all-right spherical complex $L$, the open ball 
$B\left(v, \frac{\pi}{2}\right)$ of radius $\frac{\pi}{2}$ around a vertex $v$ consists exactly of $v$ and the interior of every simplex which has $v$ as a vertex. The sphere $S\left(v, \frac{\pi}{2}\right)$ of radius $\frac{\pi}{2}$ is simply the simplicial link of $v$ in $L$. The following observation is a key element in Gromov's proof of (1): any locally geodesic segment in $L$ which has endpoints in $S\left(v, \frac{\pi}{2}\right)$ and intersects $B\left(v, \frac{\pi}{2}\right)$ has length at least $\pi$. We will use this to prove part (2).

It is easily verified that the condition given in (2) is necessary for $\Phi$ to be a local isometry. To prove sufficiency we use the fact (an easy consequence of Lemmas 1.4 and 1.5 of [6] ) that a cubical map $\Phi: X \rightarrow Y$ is locally isometric if, for every vertex $x \in X$, the induced map $\Phi_{x}: L k(x, X) \rightarrow L k(\Phi(x), Y)$ is $\pi$ distance preserving. A map $f: L^{\prime} \rightarrow L$ between piecewise spherical complexes is said to be $\pi$-distance preserving, if $d_{L^{\prime}}(p, q) \geq \pi$ implies $d_{L}(f(p), f(q)) \geq \pi$, for all $p, q \in L^{\prime}$.

Suppose that $x$ is a vertex of $X$ and that $\Phi_{x}$ is injective with image a full subcomplex $K$ of $L=L k(\Phi(x), Y)$. We show that $\Phi_{x}$ is $\pi$-distance preserving. The argument follows that of Lemma 1.6 of [6]. Given $p, q \in K$ such that $d_{L}(p, q)<\pi$ there exists a geodesic segment $\gamma \in L$ joining $p$ to $q$. (Note that links in a $\operatorname{CAT}(0)$ polyhedral complex are $\pi$-geodesic [5]). It is enough to show that $\gamma$ lies wholly in $K$. Suppose that $\gamma$ enters the interior of some simplex $\sigma$ of $L$ and let $v$ denote a vertex of $\sigma$. Then $\gamma$ enters $B\left(v, \frac{\pi}{2}\right)$. Now, by the observation made above, $\gamma$ must have an endpoint $p$ in $B\left(v, \frac{\pi}{2}\right.$ ) (since otherwise it would contain a subsegment of length at least $\pi$ ). Now any simplex which contains $p$ has $v$ as a vertex, and since $p$ lies in the subcomplex $K$, we must have $v \in K$. Since this is true for every vertex of $\sigma$, and $K$ is a full subcomplex, we deduce that $\sigma$ is a simplex of $K$. But that is to say that $\gamma$ never enters the interior of a simplex which does not already lie in $K$, and so $\gamma$ lies wholly in $K$.

\section{$3 \quad$ Graph braid groups and right-angled Artin groups}

Let $G$ be a finite graph, viewed as a simplicial complex. For each point $x \in G$ denote by $c(x)$ the carrier of $x$, that is the smallest dimensional simplex which contains $x$. Thus $c(x)$ denotes either a vertex or a (closed) edge of $G$. For

$n \in \mathbb{N}$, we define $\widetilde{X}_{n}(G)$ to be the subspace of $G^{n}$ consisting of those $n$-tuples $\left(x_{1}, x_{2}, \ldots, x_{n}\right)$ for which the $x_{i}$ have mutually disjoint carriers: $c\left(x_{i}\right) \cap c\left(x_{j}\right)=$ $\emptyset$ for $i \neq j$. This is, in fact, a subspace of the ordered $n$-point configuration space of $G$. The symmetric group $S_{n}$ acts freely and properly discontinuously on $\widetilde{X}_{n}(G)$ by permutations of the coordinates, and the quotient space may be 
viewed as a subspace of the unordered $n$-point configuration space of $G$ and is written

$$
X_{n}(G)=\widetilde{X}_{n}(G) / S_{n}=\left\{\left\{x_{1}, x_{2}, \ldots, x_{n}\right\}: c\left(x_{i}\right) \cap c\left(x_{j}\right)=\emptyset \text { for } i \neq j\right\} .
$$

Following Abrams [1, 2] we refer to $X_{n}(G)$ (respectively $\widetilde{X}_{n}(G)$ ) as the reduced unordered (respectively ordered) $n$-point configuration space of $G$. We concern ourselves here with the reduced braid group of $G$, namely

$$
R B_{n}(G)=\pi_{1}\left(X_{n}\right) .
$$

(This group contains the reduced pure braid group $R P B_{n}(G)=\pi_{1}\left(\widetilde{X}_{n}(G)\right.$ ) as an index $n$ ! subgroup).

Note that these definitions depend upon the simplicial structure on $G$, while the usual configuration spaces of $G$ do not. It was proved in [2, 3] that, after subdividing the edges of the graph $G$ sufficiently often, creating some valencetwo vertices, the reduced configuration space $X_{n}(G)$ becomes a deformation retract of the usual configuration space of $G$, and hence that the reduced braid and reduced pure braid groups coincide with the usual braid and pure braid groups, $B_{n}(G)$ and $P B_{n}(G)$, respectively. Thus the reader should keep in mind that our results about $R B_{n}(G)$ hold in particular for the full graph braid groups $B_{n}(G)$.

The reduced configuration space $X_{n}(G)$ has the structure of a cubed complex, in a very natural way. The $k$-dimensional cubes are in 1-1 correspondence with collections $\left\{c_{1}, c_{2}, \ldots, c_{n}\right\}$ of mutually disjoint simplices in $G, k$ of which are edges and $n-k$ of which are vertices. The cube associated to $\left\{c_{1}, c_{2}, \ldots, c_{n}\right\}$ is simply the set of points

$$
\mathcal{C}\left\{c_{1}, c_{2}, \ldots, c_{n}\right\}=\left\{\left\{x_{1}, x_{2}, \ldots, x_{n}\right\}: x_{i} \in c_{i} \text { for all } i\right\} \cong c_{1} \times c_{2} \times \cdots \times c_{n} .
$$

In fact, it is easily checked that $X_{n}(G)$ is a cubical complex (in the language of [5]) - each cube is embedded and the intersection of any two cubes is either empty or a single face of some dimension. It is also an almost immediate consequence of the above description of the cubical structure on $X_{n}(G)$ that all vertex links are flag complexes, and hence that $X_{n}(G)$ is a locally $\operatorname{CAT}(0)$ cubical complex as first proved by Abrams [2, 1]. In this paper we construct a locally isometric cubical map from $X_{n}(G)$ to a known CAT(0) cubed complex whose fundamental group is a right-angled Artin group. In this way, we prove that every reduced graph braid group embeds in a right-angled Artin group. 
Definition (Right-angled Artin group/graph group) Let $\Delta$ denote a finite simple graph (that is a finite graph with no loops or multiple edges). Let $\mathcal{V}$ and $\mathcal{E}$ denote the vertex and edge sets of $\Delta$ respectively. Associated to each such graph $\Delta$ is the right angled Artin group, or graph group

$$
\left.A_{\Delta}=\langle\mathcal{V}| u v=v u \text { if }(u, v) \in \mathcal{E}\right\rangle .
$$

We shall now describe a locally CAT(0) cubed complex which is an EilenbergMacLane complex for $A_{\Delta}$. This construction is well-known, and plays a crucial role for instance in the paper of Bestvina and Brady [4] on finiteness properties of groups.

The standard $n$-torus $T^{n}=\mathbb{E}^{n} / \mathbb{Z}^{n}$ may be described as the unit Euclidean cube $[0,1]^{n}$ with opposite faces identified, and so is a cubed complex with one vertex, $n$ edges and precisely one $k$-cube spanned by each set of $k$ distinct edges. To each finite set $S$ of size $n$ we may associate such a cubed $n$-torus $T(S)$ by labelling the edges by the elements of $S$. We shall also orient all edges.

Given the graph $\Delta$ we now define the cubed complex $\mathcal{T}_{\Delta}$ which is obtained from $T(\mathcal{V})$ by deleting the interior of every cube which correspond to a set of vertices in $\Delta$ which does not span a complete graph. Alternatively,

$$
\mathcal{T}_{\Delta}=(\coprod\{T(U): U \subset \mathcal{V} \text { spans a complete graph in } \Delta\}) / \sim
$$

where the $\sim$ denotes identification of $T(Q)$ as a subcomplex of $T(R)$ in the obvious way whenever $Q \subset R$. Note that $\mathcal{T}(\Delta)$ has a unique vertex, the "0-cube" $T(\emptyset)$. The link of this vertex, which we shall denote $L_{\Delta}$, has two vertices $v^{+}$and $v^{-}$for each $v \in \mathcal{V}$ (corresponding to where the edge $v$ leaves and enters the vertex). A set $\left\{v_{0}^{\varepsilon_{0}}, v_{1}^{\varepsilon_{1}}, \ldots, v_{k}^{\varepsilon_{k}}\right\}$ of $k$ distinct vertices (with $\left.\varepsilon_{i} \in\{ \pm\}\right)$ spans a $k$-simplex in $L_{\Delta}$ if and only if $v_{0}, \ldots, v_{k}$ span a complete graph in $\Delta$.

It is now obvious that $L_{\Delta}$ is a flag complex, and so by Theorem $1(1)$ that $\mathcal{T}_{\Delta}$ is a locally $\operatorname{CAT}(0)$ cubed complex. Moreover, it is clear that $\pi_{1}\left(\mathcal{T}_{\Delta}\right) \simeq A_{\Delta}$ where the standard generators of $A_{\Delta}$ are simply represented by the corresponding labelled oriented edges of $\mathcal{T}_{\Delta}$.

We return now to our finite graph $G$, and define a new graph $\Delta(G)$ whose vertex set is just the set $\{\bar{x}: x$ an edge of $G\}$ and where two vertices are joined by an edge in $\Delta(G)$ whenever the corresponding edges in $G$ are disjoint (as closed sets). This definition immediately allows the definition of a cubical map $\Phi: X_{n}(G) \rightarrow \mathcal{T}_{\Delta(G)}$ as follows. Choose an orientation for each edge in $G$, which naturally induces an orientation on each edge of $X_{n}(G)$. Recall that a $k$-cube 
of $X_{n}(G)$ is determined uniquely by a set $\left\{x_{1}, \ldots, x_{k}, u_{1}, \ldots, u_{n-k}\right\}$ of mutually disjoint simplices of $G$ where the $x_{i}$ are edges and the $u_{i}$ are vertices. The map $\Phi$ is defined by sending the cube $\mathcal{C}\left\{x_{1}, \ldots, x_{k}, u_{1}, \ldots, u_{n-k}\right\}$ onto the cube in $\mathcal{T}_{\Delta(G)}$ spanned by the edges $\bar{x}_{1}, \ldots, \bar{x}_{k}$ while respecting the orientations of all edges.
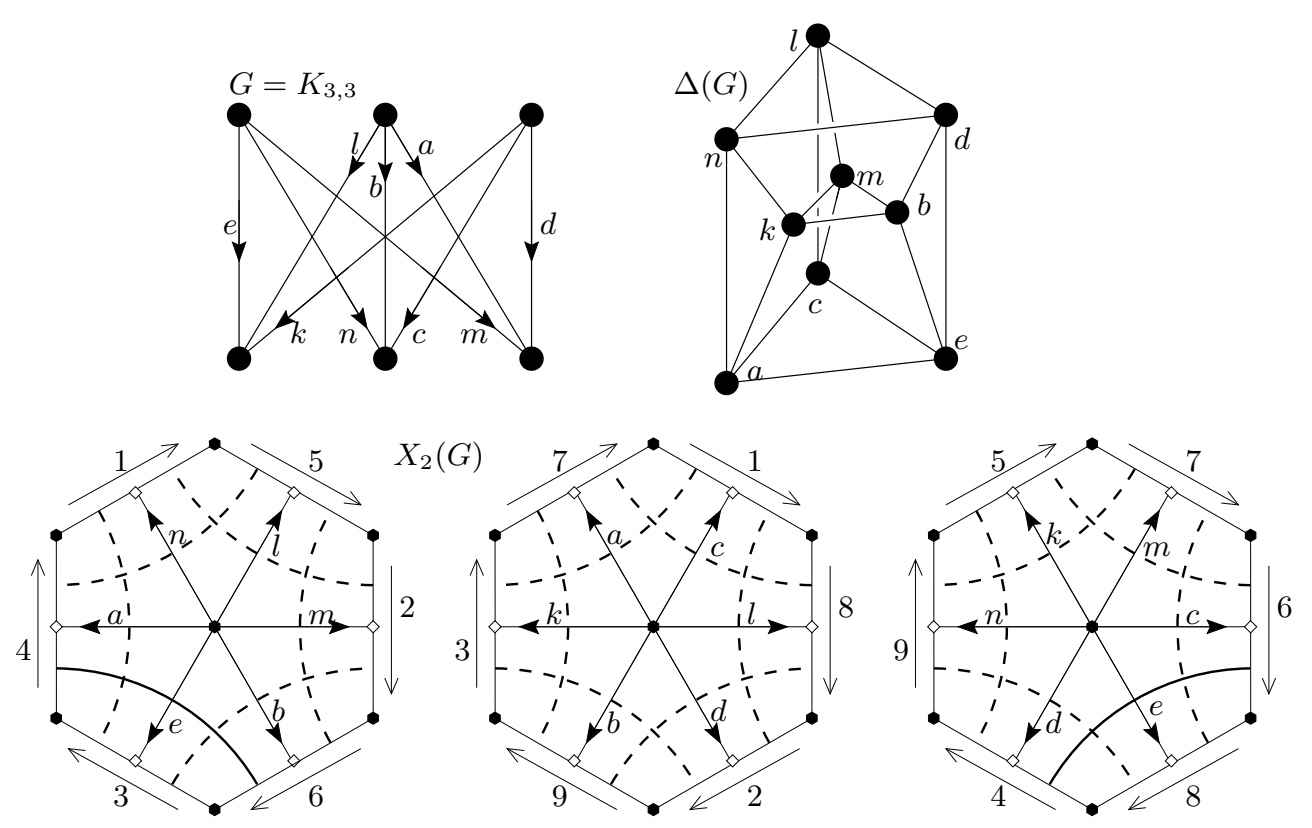

Figure 1: The reduced 2-point configuration space $X_{2}\left(K_{3,3}\right)$ of the $K_{3,3}$-graph is a nonorientable surface of Euler characteristic -3, with a cubing by 18 squares (here grouped into three hexagons, which are glued according to the arrows labelled 1 to 9 ). Its fundamental group embeds in the right-angled Artin group $A_{\Delta(G)}$.

Theorem 2 Let $G$ be any finite graph, and $n \in \mathbb{N}$. Then the map $\Phi: X_{n}(G)$ $\rightarrow \mathcal{T}_{\Delta(G)}$ described above is a local isometry and hence $\pi_{1}$-injective. Thus the reduced graph braid group $R B_{n}(G)$ embeds in the right-angled Artin group $A_{\Delta(G)}$.

Proof For simplicity write $X=X_{n}(G)$. Since we know that $\mathcal{T}_{\Delta(G)}$ is locally CAT(0) it suffices, by Theorem 1, to show that the link of each vertex of $X$ is mapped injectively onto a full subcomplex of $L_{\Delta(G)}$. A vertex of $X$ is just a set $U$ of $n$ distinct vertices of $G$. Each vertex in the link $L k(U, X)$ is uniquely determined by an edge $e$ in $G$, precisely one of whose vertices, $u$ say, lies in $U$. This link vertex shall be written $e^{+}$or $e^{-}$depending on whether $e$ happens to 
be oriented away from or into the graph vertex $u$. Now the map induced by $\Phi$ sends a vertex $e^{\varepsilon}$ in $L k(U, X)$ to the vertex $\bar{e}^{\varepsilon}$ in $L_{\Delta}$ (where $\left.\varepsilon \in\{+,-\}\right)$ and so is clearly injective on vertices. Moreover one easily observes that $e_{1}^{\varepsilon_{1}}, \ldots, e_{n}^{\varepsilon_{n}}$ span a simplex in $L k(U, X)$ if and only if $e_{1}, \ldots, e_{n}$ are mutually disjoint, if and only if $\bar{e}_{1}, \ldots, \bar{e}_{n}$ span a complete graph in $\Delta(G)$, if and only if $\bar{e}_{1}^{\varepsilon_{1}}, \ldots, \bar{e}_{n}^{\varepsilon_{n}}$ span a simplex in $L_{\Delta(G)}$. Thus the image of $L k(U, X)$ is indeed a full subcomplex of $L_{\Delta(G)}$.

We illustrate this Theorem with two examples which will be used in the next Section. The first example is the case where $G$ is taken to be the bi-partite graph $K_{3,3}$, and $n=2$. The graph $\Delta\left(K_{3,3}\right)$ and the complex $X_{2}\left(K_{3,3}\right)$ which is in fact a squaring of the closed nonorientable surface of Euler characteristic -3 , are shown in Figure 1. The dashed lines dual to the squaring together with the labels $a, b, \ldots, m, n$ constitute a "cellular dissection" of the surface, as defined in Section 4 below, and describe the embedding of $R B_{2}\left(K_{3,3}\right)$ (the fundamental group of the surface) into $A_{\Delta\left(K_{3,3}\right)}$.

The second example is the case where $G=K_{5}$, the complete graph on 5 vertices, and again $n=2$. We leave the reader to verify that in fact $X_{2}\left(K_{5}\right)$ is a squaring of the closed nonorientable surface of Euler characteristic -5 with 10 vertices all of which have valence 6 (there are therefore 15 faces and 30 edges). The graph $\Delta\left(K_{5}\right)$ is in fact the image of the 1-skeleton of the boundary of the dodecahedron under the 2 -fold quotient defined by the antipodal map.

\section{Surface groups in right-angled Artin groups}

In this section, we classify the surface groups which can be embedded in a rightangled Artin group. Let $R P^{2}$ denote the real projective plane, $K l$ the Klein bottle, and $S_{-1}$ the non-orientable surface of Euler characteristic -1 .

Theorem 3 Let $S$ be a surface.

(i) If $S \neq R P^{2}, K l$, or $S_{-1}$, then there exists a right-angled Artin group $A$ and a (quasi-isometric) embedding of $\pi_{1}(S)$ as a subgroup of $A$.

(ii) If $S=R P^{2}, K l$, or $S_{-1}$, then $\pi_{1}(S)$ does not embed as a subgroup of any right-angled Artin group.

Proof Note that the torus group $\mathbb{Z} \times \mathbb{Z}$ is already a right-angled Artin group, so embeds in one. Similary, the fundamental group of any non-closed surface group 
is free of finite rank, so is already a right-angled Artin group. In Proposition [6] we shall exhibit quasi-isometric embeddings for each closed surface of Euler characteristic less than or equal to -2 . The fundamental group of the 2 -sphere is trivial, so embeds trivially.

On the other hand, $\pi_{1}\left(R P^{2}\right)$ has order 2 , so does not embed in any torsion free group. The Klein bottle group $\pi_{1}(K l)$ has generalised torsion and so is not biorderable (see for instance [24]). Therefore $\pi_{1}(K l)$ cannot embed in any right-angled Artin group since right-angled Artin groups are known to be biorderable [9] (see also Section 6). Finally it follows from Theorem[7below (see Corollary 8) that the group $\pi_{1}\left(S_{-1}\right)$ cannot be embedded in any right-angled Artin group.

In order to construct embeddings of surfaces we shall appeal once again to the techniques of Section 2 In particular, every embedding is realised geometrically by a geodesic embedding of one $\operatorname{CAT}(0)$ space in another. Consequently, the group homomorphism obtained in each case is a quasi-isometric embedding. We note that similar embeddings of surface groups in right-angled Artin groups have been obtained in $[8$. The emphasis in that paper is somewhat different however. They show that "most" right-angled Artin groups contain non-abelian surface groups but are less concerned with realising a given surface group.

Let $S$ be compact surface. Let $c$ denote an orientation preserving simple closed curve in the interior of $S$. A transverse orientation of $c$ is a choice of one or other of the two connected components of the total space of the normal bundle to $c$ in $S$, and is simply indicated by an arrow crossing the curve from one side to the other. We extend this definition in the obvious way to allow $c$ to be a boundary component of $S$, in which case a transverse orientation is either "into" or "out of" the surface, and to arbitrary intervals $(I, \partial I)$ properly embedded in $(S, \partial S)$. Transversely oriented simple closed curves, properly embedded intervals and boundary components shall be called collectively hypercurves in $S$.

Definition 4 Let $\mathcal{V}$ be a finite set, $S$ a compact surface. A $\mathcal{V}$-dissection of $S$ is a collection $\mathcal{H}$ of hypercurves in $S$ satisfying

(i) any two hypercurves of $\mathcal{H}$ are either disjoint or intersect transversely in a discrete set of points, and no more than two hypercurves meet at any one point;

(ii) as well as being transversely oriented, each hypercurve in $\mathcal{H}$ is labelled with an element of $\mathcal{V}$ in such a way that no two hypercurves which intersect have the same label. 
Note that we are interested a priori in $\mathcal{V}$-dissections of closed surfaces (in which case all hypercurves are simple closed curves). However the above definition will allow us to construct dissections of closed surfaces by decomposing them into compact pieces with boundary and dissecting these separately. Our interest in the above definition stems from the following observation:

A $\mathcal{V}$-dissection $\mathcal{H}$ of a closed surface $S$, together with a choice of basepoint $p \in S \backslash(\cup \mathcal{H})$, determines a homomorphism of $\pi_{1}(S, p)$ to a right-angled Artin group as follows. Let $\Delta(\mathcal{H})$ be the graph with vertex set $\mathcal{V}$ and edge set $\mathcal{E}$ where $(u, v) \in \mathcal{E}$ if and only if there exist a pair of intersecting hypercurves in $\mathcal{H}$ labelled $u$ and $v$ respectively. Given a loop $\gamma$ at $p$ in $S$ which is in general position with respect to $\mathcal{H}$ (in particular we require that $\gamma$ crosses only one hypercurve at a time), we define $w_{\gamma}$ to be the word in the letters $\mathcal{V} \cup \mathcal{V}^{-1}$ obtained by reading off the labels of the hypercurves traversed by $\gamma$ (the sign of each letter in $w_{\gamma}$ is determined by whether $\gamma$ traverses the hyperplane with or against the transverse orientation). Then the homomorphism $\psi: \pi_{1}(S, p) \rightarrow$ $A_{\Delta(\mathcal{H})}$ is well-defined by setting $\psi([\gamma])=w_{\gamma}$.

A necessary condition for $\psi$ to be injective is that $\mathcal{H}$ be cellular:

Definition 5 We say that a dissection $\mathcal{H}$ of $S$ is cellular if $S \backslash(\cup \mathcal{H})$ is a disjoint union of open disks.

In this case, $\psi$ is induced by a cubical map. If the dissection $\mathcal{H}$ is cellular, then it determines in an obvious way a polyhedral decomposition of $S$. We consider the squaring of $S$ which is dual to this decomposition, and which we denote $X(S, \mathcal{H})$. Moreover, each edge of $X(S, \mathcal{H})$ is naturally labelled with orientation by an element of $\mathcal{V}$ according to the label and transverse orientation of the hypercurve to which it is dual. Opposite edges of a square in $X(S, \mathcal{H})$ are labelled similarly, and edges labelled $u$ and $v$ span a square only if $(u, v)$ is an edge of the graph $\Delta(\mathcal{H})$. Thus there is a uniquely defined cubical map $\Psi: X(S, \mathcal{H}) \rightarrow \mathcal{T}_{\Delta(\mathcal{H})}$ which sends each edge in $X(S, \mathcal{H})$ to the unique edge in $\mathcal{T}_{\Delta(\mathcal{H})}$ with the same label (respecting orientations). Evidently $\Psi$ induces $\psi$ at the level of fundamental groups.

We consider now a closed surface $S$ of Euler characteristic $\chi(S)<-1$. We shall describe a cellular dissection $\mathcal{H}$ of $S$ for which the map $\Psi: X(S, \mathcal{H}) \rightarrow \mathcal{T}_{\Delta(\mathcal{H})}$ is locally isometric. There are several cases to consider:

Case $\chi(S) \equiv 0 \bmod 2$ Let $\mathcal{V}$ be the set $\{a, b, c, d, e\}$ and consider the $\mathcal{V}$ dissected surfaces $Y_{2}$ and $Y_{2}^{\prime}$ illustrated in figure 2, Note that these dissections 


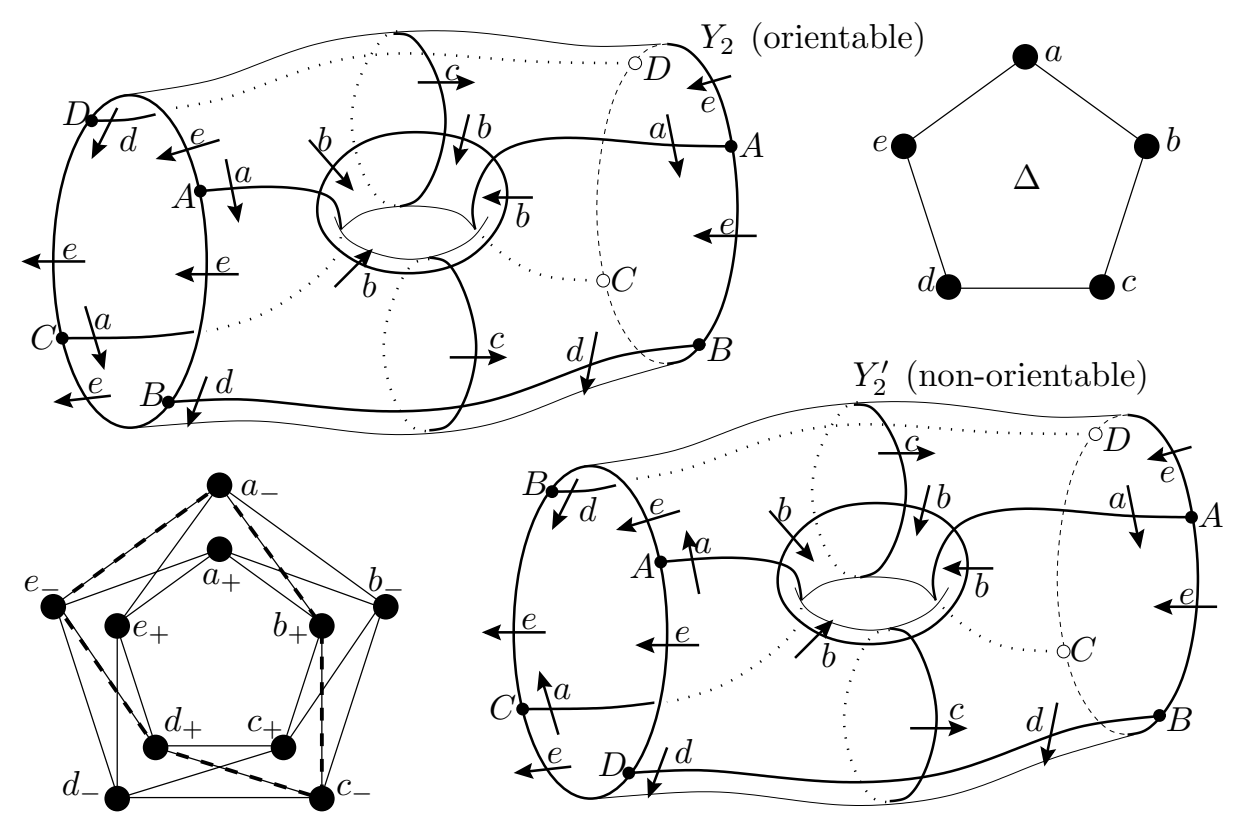

Figure 2: All surfaces $S$ with even negative Euler characteristic can be obtained by glueing copies of $Y_{2}$ and $Y_{2}^{\prime}$. The cubical decomposition $X(S, \mathcal{H})$ of $S$ contains one vertex in each component of $S \backslash(\cup \mathcal{H})$, and its link is a 5-cycle. The groups $\pi_{1}(S)$ all embed in $A_{\Delta}$, where $\Delta$ is as shown. The link $L_{\Delta}$ of the vertex of $\mathcal{T}_{\Delta}$ is a graph with 10 vertices and 20 edges, and contains the image of the link of any vertex of $X(S, \mathcal{H})$ as a full subgraph.

are cellular. Also $\chi\left(Y_{2}\right)=\chi\left(Y_{2}^{\prime}\right)=-2$. Identification of the two boundary components of $Y_{2}$ while respecting the labelling of the vertices $A, B, C, D$ as shown yields a $\mathcal{V}$-dissection of the closed orientable surface of Euler characteristic -2 . A similar operation on $Y_{2}^{\prime}$ yields cellular $\mathcal{V}$-dissection of the closed non-orientable surface of Euler characteristic -2 . The graph $\Delta$ associated to each of these dissections is the cycle of length 5 with vertices labelled as in Figure 2. It is easily checked that the cubical map $\Psi$ sending the dual squaring into $\mathcal{T}_{\Delta}$ satisfies, in each case, the conditions of Theorem 1 (2), and so is a local isometry. (The link of each vertex in the squared surface is a 5-cycle with vertices labelled $a^{ \pm}, b^{ \pm}, c^{ \pm}, d^{ \pm}, e^{ \pm}$in that order around the cycle).

More generally, we can glue $k$ copies of $Y_{2}$ end to end in a cycle (while always respecting the labelling of vertices $A, B, C, D)$ to construct a $\mathcal{V}$-dissection $\mathcal{H}$ of the orientable surface $S$ of Euler characteristic $-2 k$ for any $k \geq 1$. By the argument just given, the cubical map $\Psi: X(S, \mathcal{H}) \rightarrow \mathcal{T}_{\Delta(\mathcal{H})}$ is locally isometric. To obtain a similar result for the non-orientable surface of Euler characteristic 
$-2 k$ we simply replace one instance of $Y_{2}$ with a copy of $Y_{2}^{\prime}$ in the above construction.
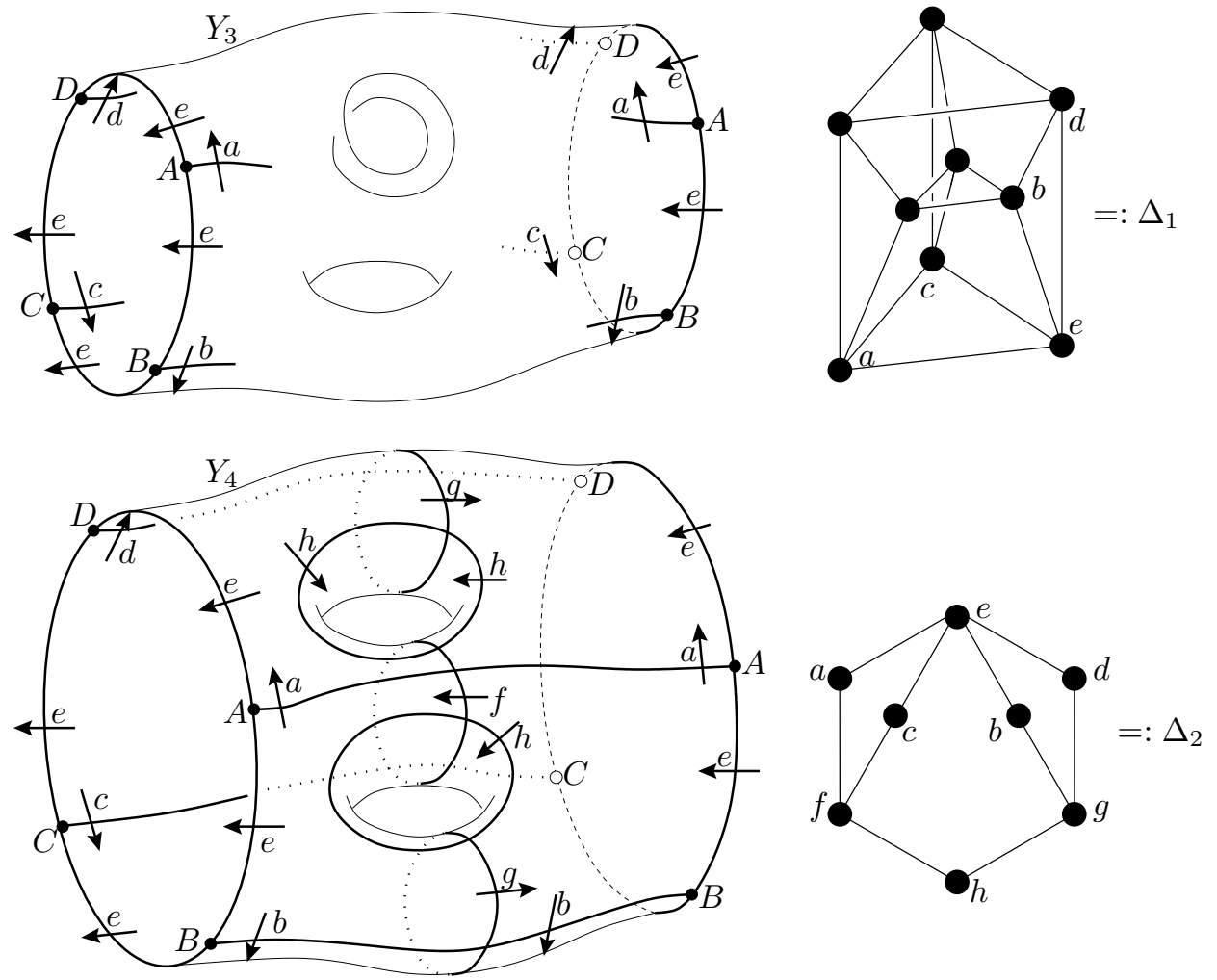

Figure 3: All surfaces $S$ with $\chi(S) \equiv-3 \bmod 4$, where $\chi(S) \leqslant-3$, and with $\chi(S) \equiv$ $-1 \bmod 4$, where $\chi(S) \leqslant-9$, can be obtained by glueing copies of $Y_{3}$ and $Y_{4}$. Their fundamental groups embed in the right-angled Artin group $\mathcal{I}_{\Delta(\mathcal{H})}$, where $\Delta(\mathcal{H})$ is the graph obtained by identifying the vertices $a, b, c, d$ and $e$, and the edges connecting them, in $\Delta_{1}$ and $\Delta_{2}$.

Case $\chi(S) \equiv-3 \bmod 4$ Recall that the the cubical complex $X_{2}\left(K_{3,3}\right)$ is simply the squaring of the closed surface $S_{-3}$ of Euler characteristic -3 illustrated in Figure 1. Dual to this is a $\mathcal{V}_{1}$-dissection $\mathcal{H}_{1}$ of the closed surface (also illustrated in Figure 1), where $\mathcal{V}_{1}=\{a, b, c, d, e, k, l, m, n\}$, and where $\Delta\left(\mathcal{H}_{1}\right)=\Delta\left(K_{3,3}\right)$ is the graph $\Delta_{1}$ shown in Figure 3 . Note that every hypercurve of $\mathcal{H}_{1}$ has edge-length 4 (as measured in the polygonal decomposition of $S_{-3}$ associated to this dissection). Also, there are no two hypercurves with the same label. Now cut $S_{-3}$ open along the hypercurve labelled $e$ to obtain a 
surface with two boundary components whose vertices are labelled $A, B, C, D$ as in Figure 3. This surface, which we denote $Y_{3}$, carries a naturally induced $\mathcal{V}_{1}$-dissection. Consider also the $\mathcal{V}_{2}$-dissected surface $Y_{4}$ shown in Figure 3 where $\mathcal{V}_{2}=\{a, b, c, d, e, f, g, h\}$ are the vertices of the associated graph $\Delta_{2}$ shown in the figure. Let $\mathcal{V}=\mathcal{V}_{1} \cup \mathcal{V}_{2}$, where the labels $a, b, c, d, e$ are the only elements common to $\mathcal{V}_{1}$ and $\mathcal{V}_{2}$.

Now gluing $k$ copies of $Y_{4}$ and one copy of $Y_{3}$ end to end in a cycle (while respecting, of course, the transverse orientations on the boundary components as well as the vertex labels $A, B, C, D)$ results in a cellular $\mathcal{V}$-dissection $\mathcal{H}$ of the surface $S$ of Euler characteristic $-(3+4 k)$, for $k \geq 0$. It is again easily seen that the cubical map $\Psi: X(S, \mathcal{H}) \rightarrow \mathcal{T}_{\Delta(\mathcal{H})}$ is locally isometric. Note that $\Delta(\mathcal{H})$ is the graph obtained by amalgamating $\Delta_{1}$ and $\Delta_{2}$ as shown in Figure 3.

Case $\chi(S) \equiv-1 \bmod 4$ We suppose $\chi(S)<-1$. The case $\chi(S)=-5$ is covered by the fact that $X_{2}\left(K_{5}\right)$ is a squaring of this surface which embeds in $\mathcal{T}_{\Delta\left(K_{5}\right)}$ via a locally isometric cubical map, as in Section 3. The remaining cases $\chi(S) \leq-9$ may be treated exactly as in the previous paragraph by gluing together 3 copies of $Y_{3}$ and $k$ copies of $Y_{4}$ to obtain a cellular dissection of the surface of Euler characteristic $-(9+4 k)$, for $k \geq 0$.

Combining the three preceding paragraphs gives:

Proposition 6 For each closed surface $S$ other than $R P^{2}, K l$, and $S_{-1}$, there exists a squaring $X(S)$ of $S$, a graph $\Delta$ (without loops or multiple edges), and a locally isometric cubical map $\Psi: X(S) \rightarrow \mathcal{T}_{\Delta}$, inducing an injection $\psi: \pi_{1}(S) \rightarrow A_{\Delta}$ on the level of fundamental groups.

We turn now to consider the surface $S_{-1}$ of Euler characteristic -1 , whose fundamental group admits the following presentation:

$$
\pi_{1}\left(S_{-1}\right)=\left\langle X, Y, Z \mid X^{2} Y^{2}=Z^{2}\right\rangle .
$$

The following Theorem generalises a well-known Theorem of Lyndon [18 for free groups. See [19] for a more complete discussion of the equation $x^{2} y^{2}=z^{2}$ over a free group.

Theorem 7 Let $A$ denote an arbitrary right-angled Artin group. If elements $x, y, z \in A$ satisfy the relation $x^{2} y^{2}=z^{2}$ in $A$ then $x, y$ and $z$ are mutually commuting elements. 
Proof We will use the fact that the relation $x^{2} y^{2}=z^{2}$ in $A$ implies the existence of a homomorphism $\varphi: \pi_{1}\left(S_{-1}\right) \rightarrow A$ such that $\varphi(X)=x, \varphi(Y)=y$ and $\varphi(Z)=z$. In the preceding paragraphs we constructed homomorphisms from surface groups to right-angled Artin groups by producing dissections of the surface. The key observation at this point is that this method is completely general:

Claim A Let $S$ be a closed surface, and $A$ a right-angled Artin group. Every homomorphism $\psi: \pi_{1}(S) \rightarrow A$ is defined by a dissection of $S$.

Proof of Claim Consider a polygonal decomposition of the surface $S$ with one vertex and one 2-cell $D$, and orient the edges. This gives a 1-relator presentation $\mathcal{P}$ of the closed surface group $\pi_{1}(S)$ where the generators correspond to the labelled edges and the relator word can be read around the boundary of the polygon $D$. (e.g: the presentation already given above for $\pi_{1}\left(S_{-1}\right)$ comes from description of $S_{-1}$ as a hexagon with face pairings).

Let $\mathcal{V}$ denote the standard generating set of $A$ (the vertex set of the defining graph $\Delta$ ). For each generator $U$ of $\mathcal{P}$ choose a word in the letters $\mathcal{V}$ and their inverses which represents $\psi(U)$ (and which, by abuse of notation, we shall also denote by $U$ ). Now subdivide the boundary of the polygon $D$ so that its sides are labelled now by the words $X, Y, Z, \ldots$, the new edges being oriented and labelled by the letters of $\mathcal{V}$.

We now show how to construct a dissection of the surface $D$ which is consistent with the boundary labelling: that is to say that (i) the endpoints of those hypercurves which are properly embedded arcs lie on the midpoints of edges of $\partial D$ and the labelling and tranverse orientation of each such hypercurve agrees with the edge labelling at each of its endpoints, and (ii) every edge in $\partial D$ is at the endpoint of exactly one hypercurve.

The boundary word $W_{0}$ which is read around the relator disk $D\left(W_{0}=\right.$ $X^{2} Y^{2} Z^{-2}$ in the case $S_{-1}$ ) represents the identity in $A$. There is therefore a sequence $W_{0}, W_{1}, W_{2}, \ldots, W_{n}=1$ of words in $\mathcal{V} \cup \mathcal{V}^{-1}$ such that $W_{i}$ is obtained from $W_{i-1}$ by either (i) a trivial insertion of $u u^{-1}$ or $u^{-1} u$ (for $u \in \mathcal{V}$ ), or (ii) a trivial deletion, or (iii) a substitution of a subword $u^{\epsilon} v^{\epsilon^{\prime}}$ of $W_{i}\left(\epsilon, \epsilon^{\prime} \in\{ \pm 1\}\right)$ with the word $v^{\epsilon^{\prime}} u^{\epsilon}$ in the case that $(u, v)$ is an edge of the defining graph $\Delta$. Decompose $D$ as the union of a disk $D^{\prime}$ in the interior of $D$ and an annulus $A$, and label the boundary of $D^{\prime}$ by the word $W_{1}$. By induction on the number of steps of type (i)-(iii) needed to transform a word into the trivial word, we may suppose that $D^{\prime}$ admits a dissection which is consistent with the labelling 

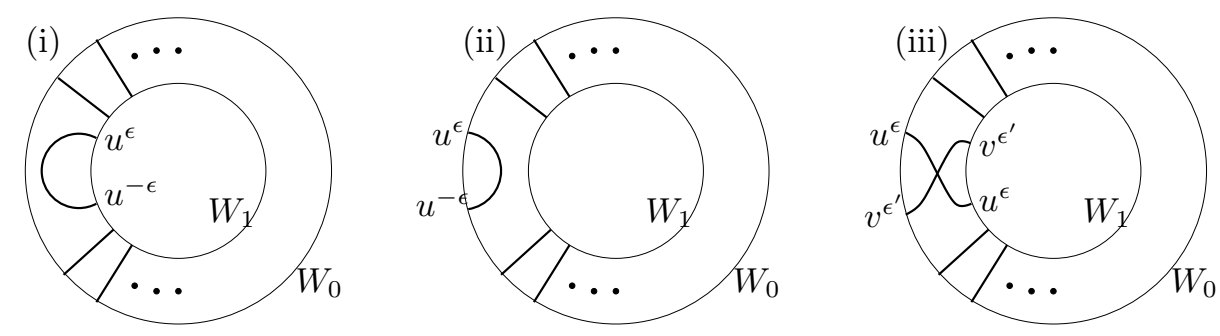

Figure 4: Extending the dissection of $D^{\prime}$ across the annulus $A$ to a dissection of $D=D^{\prime} \cup A$.

$W_{1}$ of the boundary. This dissection extends across the annulus $A$ as shown in Figure 4 (i), (ii) or (iii), according to whether the transformation from $W_{0}$ to $W_{1}$ is of type (i), (ii), or (iii) respectively, to give the desired dissection of the disk $D$.

Having shown that there exists a dissection of $D$ consistent with the boundary labels, it remains to observe that such a dissection induces a dissection of the surface $S$ (after pairwise identification of the edges of the polygon $D)$. Moreover, this dissection of $S$ clearly induces the given homomorphism $\psi: \pi_{1}(S) \rightarrow A$, as required.

Note that the definition of the homomorphism $\psi$ by a dissection $\mathcal{H}$ of a closed surface $S$ actually depends on a choice of basepoint $*$ in $S$. In the above argument, the basepoint is simply just the vertex of the polygonal decomposition of $S$. Allowing the basepoint to move with respect to the dissection $\mathcal{H}$ only changes the definition of $\psi$ up to an inner automorphism of the image group $A$. We say that homomorphisms $\psi$ and $\psi^{\prime}$ from $\pi_{1}(S)$ to $A$ are equivalent if there exists $g \in A$ such that $\psi^{\prime}(w)=g \psi(w) g^{-1}$ for all $w \in \pi_{1}(S)$.

Claim B Let $\mathcal{H}$ be a dissection of $S$ inducing the homomorphism $\psi$. The following modifications of $\mathcal{H}$ do not change the equivalence class of $\psi$.

(i) Removing from $\mathcal{H}$ any or all hypercurves which are homotopically trivial in $S$;

(ii) Changing $\mathcal{H}$ to a different dissection $\mathcal{H}^{\prime}$ by a homotopy of one or more hypercurves in $\mathcal{H}$ without changing labels or orientations and without introducing any intersection between hypercurves which are already disjoint in $\mathcal{H}$. 
Proof of Claim (i) Let $c$ be a homotopically trivial hypercurve of $\mathcal{H}$. Then $c$ bounds a disk $B$ in $S$. Up to equivalence of $\psi$ we are free to choose the basepoint of $S$ to lie outside $B$. Now observe that any element of $\pi_{1}(S)$ may be represented by a loop which is disjoint from from $B$, so that the curve $c$ does not enter at all into the definition of $\psi$ and may be removed.

(ii) We leave part (ii) as an exercise for the reader.

We now return to the homomorphism $\varphi: \pi_{1}\left(S_{-1}\right) \rightarrow A$ introduced at the beginning of the proof. Theorem 7 is now proved by showing that $\varphi\left(X Y Z^{-1}\right)=$ 1. For then we have relations $x y=z$ and $x^{2} y^{2}=z^{2}$ in $A$, from which it easily follows that $x y=y x$, and moreover that both $x$ and $y$ commute also with $z$. (Note that this proof only depends on the equivalence class of $\varphi$ ).

Choose a simple loop $\gamma$ at the basepoint in $S_{-1}$ which represents the element $X Y Z^{-1}$. We make the following important observation:

Claim C Any homotopically nontrivial orientation preserving simple closed curve $c$ in $S_{-1}$ which intersects $\gamma$ transversely must intersect $\gamma$ in exactly two points and must bound a Möbius band in $S_{-1}$.

Proof of Claim We cut $S_{-1}$ open along the simple closed curve $\gamma$. Since $\gamma$ is an orientation reversing curve in $S_{-1}$, the surface $T$ resulting from this operation is homeomorphic to a torus with an open disk removed. Let $b$ denote the boundary of $T$. Then $S_{-1}$ is obtained as the quotient of $T$ by the antipodal map on $b$. The curve $c$, as viewed in $T$, consists of a collection of mutually disjoint $\operatorname{arcs} c_{1}, c_{2}, \ldots, c_{k}$ embedded in $T$ so that their endpoints lie on distinct points of $b$.

Define the simple closed curves $\lambda=S^{1} \times\{1\}, \mu=\{1\} \times S^{1}$ and $\delta=\{(x, x)$ : $\left.x \in S^{1}\right\}$ in the torus $S^{1} \times S^{1}$. These curves are disjoint except at the common point $(1,1)$. Suppose now that $T$ is obtained from $S^{1} \times S^{1}$ by deleting an open neighbourhood of $(1,1)$, and let $l, m, d$ denote the arcs in $T$ obtained by intersecting $\lambda, \mu$ and $\delta$ respectively with $T$.

Up to a homeomorphism of $T$ we may suppose that each of the mutually disjoint $\operatorname{arcs} c_{1}, \ldots, c_{k}$ is parallel to one of $l, m$, or $d$, and that at least one of them is parallel to $l$. Re-label these $\operatorname{arcs} l_{1}, \ldots, l_{r}, d_{r+1}, \ldots, d_{s}, m_{s+1}, \ldots, m_{k}$ accordingly (where $1 \leq r \leq s \leq k$ ). We label the endpoints of an $\operatorname{arc} \alpha$ by $\alpha^{+}$ 
and $\alpha^{-}$. Now observe that, up to re-numbering and re-orienting of the arcs, their endpoints appear in the following cyclic order around $b$ :

$$
\begin{array}{r}
l_{1}^{+}, l_{2}^{+}, \ldots, l_{r}^{+}, d_{r+1}^{+}, \ldots, d_{s}^{+}, m_{s+1}^{+}, \ldots, m_{k}^{+}, l_{r}^{-}, \ldots, l_{2}^{-}, l_{1}^{-}, d_{s}^{-}, \ldots, \\
d_{r+1}^{-}, m_{k}^{-}, \ldots, m_{s+1}^{-} .
\end{array}
$$

Since $c$ is formed from these arcs by identifying pairs of vertices which are antipodal in this cyclic order, the arcs $l_{1}$ and $l_{r}$ (or in the case $r=1$, just the arc $l_{1}$ by itself) form a connected component of $c$. But since $c$ is connected and orientation preserving we must have $r=s=k=2$ and $c$ consists of just $l_{1} \cup l_{2}$. Moreover, since $l_{1}$ and $l_{2}$ are parallel in $T, c$ bounds a Möbius band in $S_{-1}$.

Proof that $\varphi\left(X Y Z^{-1}\right)=1$ We may clearly consider $\varphi$ up to equivalence. By Claim A, $\varphi$ may be defined by a dissection $\mathcal{H}$ of $S_{-1}$. By Claim B(i) we may suppose that every hypercurve of $\mathcal{H}$ is homotopically non-trivial. We may suppose also that the loop $\gamma$ which represents $X Y Z^{-1}$ is transverse to $\mathcal{H}$. Let $c \in \mathcal{H}$ be a hypercurve which intersects $\gamma$ in at least one point. Then, by Claim C, $c$ bounds a Möbius band $M$ and intersects $\gamma$ in two points. By choosing an innermost such hypercurve $c$ we may suppose that $M$ does not contain any hypercurve in its interior. By a homotopy of $c$ satisfying the conditions of Claim B(ii) we may futhermore assume up to an equivalence of $\varphi$ that the following hold:

- that the basepoint $*$ of $S_{-1}$ does not lie in $M$,

- that no two hypercurves of $\mathcal{H}$ intersect on the interior of $M$, and

- that any hypercurve $c^{\prime}$ of $\mathcal{H}$ which intersects $M$ does so essentially - no component of $c^{\prime} \cap M$ cobounds a disk together with a subinterval of $c$.

As a consequence of the last two of these conditions, any loop $\alpha$ at $*$ in $S_{-1}$ is homotopic to one which does not intersect any hypercurves while in the interior of $M$. It now follows that deleting $c$ from the dissection $\mathcal{H}$ does not change the definition of $\varphi$ (up to equivalence).

By a straightforward induction we may now suppose that there are no hypercurves in $\mathcal{H}$ which intersect $\gamma$. That is to say, $\varphi([\gamma])=1$ as required. This completes the proof of Theorem [

Corollary 8 The fundamental group of the surface $S_{-1}$ of Euler characteristic -1 does not embed as a subgroup of any right-angled Artin group.

Note that the proof of Theorem 7 shows that $\pi_{1}\left(S_{-1}\right)$ is not even residually isomorphic to a subgroup of a right-angled Artin group. 


\section{The word and conjugacy problems for right-angled Artin groups}

The word and conjugacy problems for right-angled Artin groups have been widely studied and are well understood [12, 14, 15, 25, 26, 27. In this section we present what we believe to be a particularly simple explanation of the solutions as outlined in 25. We use, from the previous section, the notion of a "dissection of $D$ consistent with the boundary labelling" which is equivalent (in fact dual to) a Dehn/Van Kampen diagram. We note also that hypercurves of a dissection associated to a generator $t$ play the same role as $t$-corridors used by various authors in the study of van Kampen diagrams.

Consider a right-angled Artin group $A_{\Delta}$ with defining graph $\Delta$. The word problem in $A_{\Delta}$ is solved by the following "algorithm":

Let $w=u_{1}^{\epsilon_{1}} u_{2}^{\epsilon_{2}} \ldots u_{k}^{\epsilon_{k}}$, with $\epsilon_{i} \in\{ \pm 1\}$, be a freely reduced word in the generators of $A_{\Delta}$. We say that $w$ is $\Delta$-reduced if there exist no $1 \leq i<j \leq k$ such that $u_{i}^{\epsilon_{i}}=\left(u_{j}^{\epsilon_{j}}\right)^{-1}$ and $\left(u_{i}, u_{r}\right)$ is an edge of $\Delta$ for all $i<r<j$. Obviously, if $w$ is not $\Delta$-reduced then it may easily be simplified by removing the letters $u_{i}^{\epsilon_{i}}$ and $u_{j}^{\epsilon_{j}}$ to give a shorter word representing the same element of $A_{\Delta}$. This simplification process continues until one reaches a $\Delta$-reduced word. We claim that the element of $A_{\Delta}$ under consideration is trivial if and only if this $\Delta$-reduced word is trivial.

The proof of this last claim is as follows: suppose that $w$ is a nontrivial word that represents the identity element in $A_{\Delta}$. Let $D$ be a disk whose boundary is subdivided into an edge path labelled by the word $w$. Then, as in the proof of Claim A, we can construct a dissection of $D$ which is consistent with the boundary labelling. One can now choose a non-simple hypercurve $c$ (i.e: one which is not just a simple closed curve) which is innermost in the following sense: any other non-simple hypercurve which is disjoint from $c$ separates $c$ from the vertex on $\partial D$ which marks where the word $w$ begins and ends. It follows that the letters at the endpoints of $c$ commute with all the letters between them in the word $w$ (since $c$ must cut the corresponding hypercurves), and hence that $w$ is not $\Delta$-reduced. Thus the only $\Delta$-reduced word which represents the identity is the trivial word.

The above result may be strengthened slightly, and extended to the case of cyclic words as in Proposition 9 below (see also 25], and the remark below). A cyclically reduced word $w$ in the generators of $A_{\Delta}$ is said to be cyclically $\Delta$-reduced if no cyclic permutation of $w$ contains a subword of the form $u^{\epsilon} v_{1}^{\epsilon_{1}} \ldots v_{n}^{\epsilon_{n}} u^{-\epsilon}$ 
where $\left(u, v_{i}\right)$ is an edge of $\Delta$ for every $i=1, \ldots, n$. On the other hand, two words in the generators of $A_{\Delta}$ are said to be $\Delta$-equivalent if they are related by a finite sequence of commutations - substitution of a subword $u^{\epsilon} v^{\nu}$ by $v^{\nu} u^{\epsilon}$ under the constraint that $(u, v)$ be an edge of $\Delta$ - and are said to be cyclically $\Delta$-equivalent if they are related by a finite sequence of commutations and cyclic permutations. Note that these equivalence operations do not change the length of a word.

Proposition 9 Let $A_{\Delta}$ be the right-angled Artin group with defining graph $\Delta$.

(i) Two $\Delta$-reduced words represent the same element of $A_{\Delta}$ if and only if they are $\Delta$-equivalent.

(ii) Two cyclically $\Delta$-reduced words represent the same conjugacy class in $A_{\Delta}$ if and only if they are cyclically $\Delta$-equivalent.

Proof (i) Take $\Delta$-reduced words $x$ and $y$ representing the same element in $A_{\Delta}$, and construct a dissection consistent with boundary labelling for a disk $D$ with boundary labelled by the word $x y^{-1}$. Since $x$ and $y$ are $\Delta$-reduced we may suppose that every non-simple hypercurve joins a letter in $x$ with a similar letter in $y$. But then it is easy to see how to construct a finite sequence of commutations to show that $x$ and $y$ are $\Delta$-equivalent, given that hypercurves which cross correspond to letters which commute.

(ii) Take $\Delta$-reduced words $x$ and $y$ representing conjugate elements of $A_{\Delta}$, so $x=w y w^{-1}$ for some word $w$. Now construct a dissection consistent with boundary labelling for a disk $D$ with boundary labelled by the word $x^{-1} w y w^{-1}$, and glue together the two subsegments of $\partial D$ labelled by the word $w$ to give an annulus with boundaries labelled by the words $x$ and $y$ respectively, and which carries a dissection which is consistent with these boundary labels. Again, since $x$ and $y$ are $\Delta$-reduced, every non-simple hypercurve joins a letter in $x$ (on one side of the annulus) with a letter in $y$ (on the other side), and one easily shows that $x$ and $y$ are cyclically $\Delta$-equivalent.

Remark The second part of Proposition 9 was given by Servatius in 25. and yields the polynomial time solution to the conjugacy problem alluded to in the Introduction. The first part was stated without proof in [25]. The result (or at least the analogous result stated in terms of "expressions" and "syllables" in Section [ below) was however treated in the more general context of graphs of groups by E. R. Green in [12] (see also [14 for statements) and a 
different proof of Green's result was given in [15, using Dehn diagrams. We claim that our proofs may be easily modified to apply also in the context of graphs of groups. One simply allows each non-simple hypercurve of a dissection to be, not just an arc, but rather a properly embedded trivalent graph whose edges are transversely oriented and labelled by syllables associated to a common vertex (non-trivial elements of a common vertex group) in such a way that the product of labels read around any vertex of the hypercurve is the identity. This branching allows for "amalgamation" and "un-amalgamation" of syllables in a given expression. We leave the details to the interested reader.

\section{Right-angled Artin groups in pure surface braid groups}

The principal aim of this section is to prove the following:

Theorem 10 Let $\Delta$ be a graph without loops or multiple edges, and let $n$ denote the number of vertices in $\Delta$. Then the right-angled Artin group $A_{\Delta}$ associated to $\Delta$ can be faithfully represented as a subgroup of a pure surface braid group $P B_{n}(S)$, where $S$ is a compact surface with nonempty boundary.

Since pure surface braid groups of compact surfaces with boundary are biorderable by [10] we have as an immediate corollary that right-angled Artin groups are bi-orderable. Bi-orderability of right-angled Artin groups was previously proved by Duchamp and Thibon 9] by generalising the Magnus construction for free groups.

Theorem 10 will be eventually established by proving Proposition 13 below. We fix some notation first. Let $S$ denote a surface (not necessarily connected) which is obtained by plumbing together, in an arbitrary manner, a collection $A_{1}, A_{2}, \ldots, A_{n}$ of disjoint annuli. We make this more precise as follows.

For convenience we "coordinatise" each annulus $A_{i}$ via a homeomorphism to $\mathbb{R} / N \mathbb{Z} \times[0,1]$, where $N$ is a sufficiently large natural number. By a patch (on the annulus $A_{i}$ ) we mean a closed subset of $A_{i}=\mathbb{R} / N \mathbb{Z} \times[0,1]$ of the form $[t, t+1] \times[0,1]$ for some $t \in \mathbb{R} / N \mathbb{Z}$. The construction of $S$ depends upon

(1) a choice of a finite collection $\mathcal{P}$ of mutually disjoint patches on the annuli $A_{1}, \ldots, A_{n}$

(2) a choice of a family $\mathcal{B}$ of ordered pairs $\left(Q, Q^{\prime}\right)$ where $Q$ and $Q^{\prime}$ are elements of $\mathcal{P}$ coming from different annuli; and 
(3) for each pair $\left(Q, Q^{\prime}\right) \in \mathcal{B}$, a choice of homeomorphism $h: Q \rightarrow Q^{\prime}$ such that, if $Q=[s, s+1] \times[0,1] \subset A_{i}$ and $Q^{\prime}=[r, r+1] \times[0,1] \subset A_{j}$ say, then $h(x, y)=(f(y), g(x))$ where $f:[0,1] \rightarrow[r, r+1]$ and $g:[s, s+1] \rightarrow[0,1]$ are homeomorphisms.

Definition 11 The surface $S$ is the defined to be the identification space

$$
S=\left(\coprod_{i=1}^{n} A_{i}\right) / \approx
$$

where $\approx$ is the equivalence relation determined by the family of homeomorphisms $h: Q \rightarrow Q^{\prime}$ for $\left(Q, Q^{\prime}\right) \in \mathcal{B}$.

Given the surface $S$ defined in this way we define the graph $\Delta(S)$ as follows. The vertex set of $\Delta(S)$ is in one-to-one correspondance with the annuli of $S$ and is written $\mathcal{V}=\left\{a_{1}, a_{2}, \ldots, a_{n}\right\}$. The pair $\left(a_{i}, a_{j}\right)$ represents an edge of $\Delta(S)$ if and only if $A_{i}$ and $A_{j}$ are disjoint subsets of $S$.

Remark 12 It is clear that every graph without loops or multiple edges may be realised as $\Delta(S)$ for some surface constructed as above. Note, however, that for a given graph the construction is far from unique. In many cases a given graph may be realised by building a planar surface $S$ (which results in an embedding of the associated right-angled Artin group in a classical braid group - see Section (7). This construction requires drawing a family of simple closed curves in the plane which are mutually transverse and have the desired intersection properties. In Figure [5] we illustrate such circle drawings which realise the 5-cycle of Figure 2, the graph $\Delta\left(K_{5}\right)$, and the graph $\Delta_{1} \cup \Delta_{2}$ of Figure 3 (and in particular its full subgraph $\Delta_{1}=\Delta\left(K_{3,3}\right)$ ). The surface $S$ is obtained in each case by taking a planar neighbourhood of the union of circles. Note that the fact that the diagram of circles in Figure 5(a) has the symmetry of an 5-cycle is a consequence of the fact that the 5-cycle is self-dual. In general, one should expect a much more complicated circle diagram when $\Delta$ is an $n$-cycle for $n>5$.

We now consider the $n$-string braid group on the surface $S$ of Definition 11$]$ It will be convenient to let $p_{i}$ denote the point $(0,0)$ and $q_{i}$ the point $\left(0, \frac{1}{2}\right)$ of the $i^{\text {th }}$ annulus $A_{i}$, for each $i=1, \ldots, n$. We may suppose in the above construction that these points are not contained in any of the patches of $\mathcal{P}$. We take as our set of "marked points" the set $\mathcal{Q}=\left\{q_{i}: i=1, \ldots, n\right\}$. For $1 \leq i \leq n$, we define $\beta_{i} \in B_{n}(S)$ to be the braid that moves the marked point $q_{i}$ once around $A_{i}$ in the positive direction, and leaves all the other points in $\mathcal{Q}$ fixed. More precisely, the braid $\beta_{i}$ is represented by the path of 
$n$-tuples, $\beta_{i}(t)=\left(q_{1}, \ldots, q_{i-1}, \gamma_{i}(t), q_{i+1}, \ldots, q_{n}\right)$, where $\gamma_{i}(t)=\left(N t, \frac{1}{2}\right) \in A_{i}=$ $\mathbb{R} / N \mathbb{Z} \times[0,1]$ for $t \in[0,1]$. We observe that setting $\varphi\left(a_{i}\right)=\beta_{i}^{2}$, for each $i=1, \ldots, n$, defines a homomorphism from $A_{\Delta(S)}$ to $P B_{n}(S)$. Theorem [10] is now proved by establishing the following:
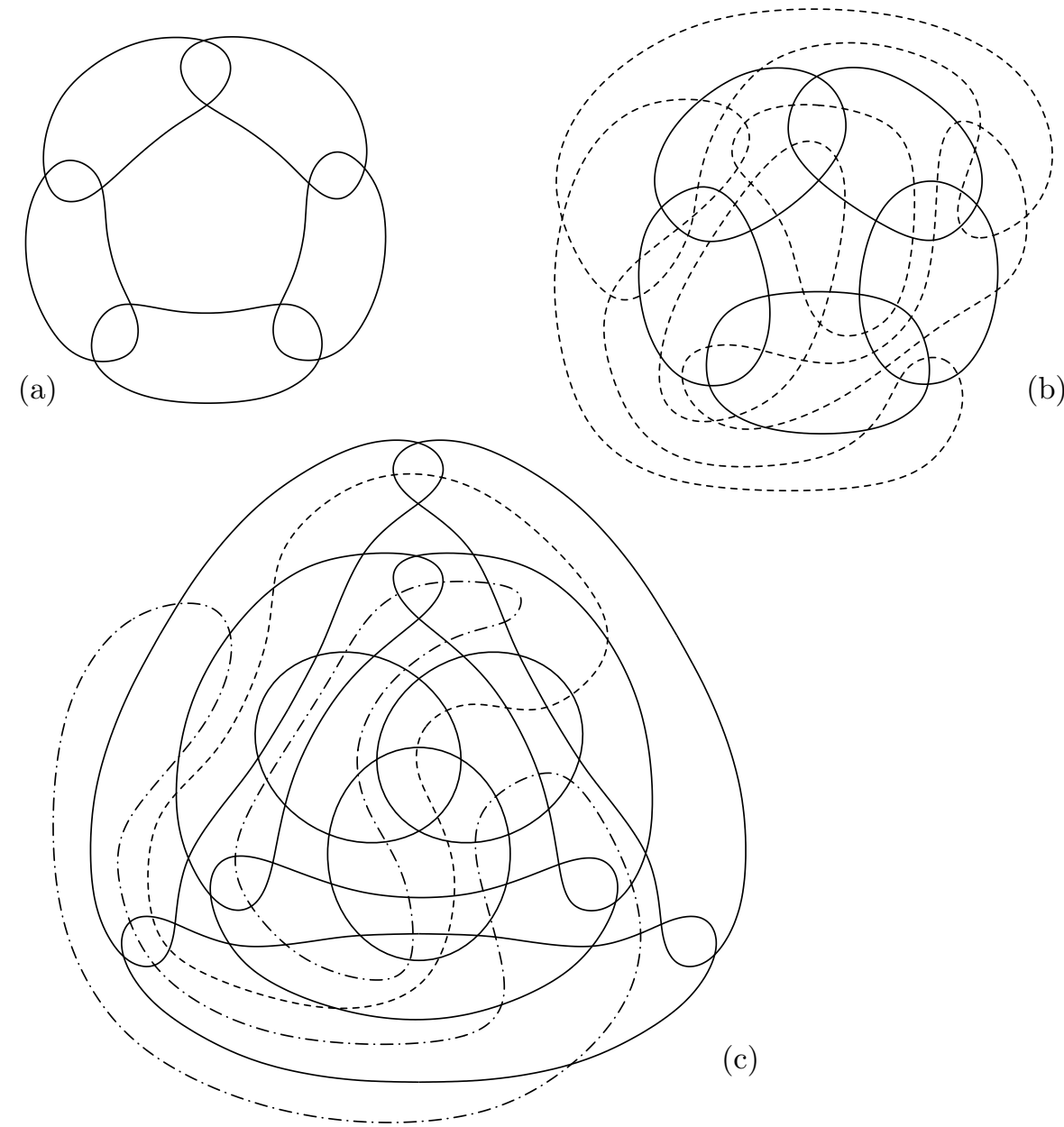

Figure 5: Circle diagrams which yield planar surfaces $S$ with $\Delta(S)$ equal to (a) the 5-cycle; (b) $\Delta\left(K_{5}\right)$; and (c) $\Delta_{1} \cup \Delta_{2}$ with the full subgraph $\Delta_{1}=\Delta\left(K_{3,3}\right)$ being realised by the plain (non-dashed) circles.

Proposition 13 Let $S$ denote any surface constructed from $n$ annuli as in Definition [1]. Then the representation $\varphi: A_{\Delta(S)} \rightarrow P B_{n}(S)$ defined by $\varphi\left(a_{i}\right)=$ $\beta_{i}^{2}$ is faithful. 
Let $F$ denote the $n$-punctured surface $S \backslash \mathcal{Q}$. We note that (pure) braids induce homeomorphisms of $F$ which are unique up to ambient isotopy of $F$ and well-defined on braid equivalence classes. Thus we have a homomorphism $\mu: P B_{n}(S) \rightarrow \mathcal{M}(F)$, where $\mathcal{M}(F)$ denotes the mapping class group of the surface $F$ relative to the boundary $\partial F=\partial S$.

By a diagram in $F$ we mean a smooth embedding $E:\{1, \ldots, n\} \times[0,1) \rightarrow F$ of $n$ disjoint half-open intervals into $F$ which may be extended to a smooth embedding $E^{\prime}:\{1, \ldots, n\} \times[0,1] \rightarrow S$ of $n$ disjoint closed intervals into $S$ by setting $E^{\prime}(i, 1)=q_{i}$. Let $E_{i}:[0,1) \rightarrow F$ denote the $i$ th curve in the diagram $E$ in $F$, that is $E_{i}(t)=E(i, t)$. We shall say that two diagrams in $F$ are equivalent if there is an ambient isotopy of $F$ relative to $\partial F$ which carries one diagram onto the other. (Note that considering ambient isotopy classes relative to the boundary means that the basepoints $p_{i}$ are always fixed while the open end of each curve in a curve diagram is effectively anchored in the vicinity of the associated puncture).

The group $P B_{n}(S)$ clearly acts on the set of equivalence classes of diagrams in $F$ via the mapping class group (i.e: via the homomorphism $\mu: P B_{n}(S) \rightarrow$ $\mathcal{M}(F))$.

We define the diagram $E^{0}$ in $S$ to consist of the $n$ straight line segments joining each point $p_{i}$ with the corresponding $q_{i}$. Thus $E^{0}(i, t)=E_{i}^{0}(t)$ is the point $\left(0, \frac{t}{2}\right)$ in $A_{i}$, for each $i=1, \ldots, n$ and each $t \in[0,1]$.

We now cut $F$ into simply connected pieces by introducing a family $\mathcal{C}$ of mutually disjoint intervals properly embedded in $(F, \partial F)$ as indicated in Figure 6 . Namely, we cut from $q_{i}$ to the point $(0,1)$ in $A_{i}$ (directly opposite $p_{i}$ ), and we cut across the annulus $A_{i}$ once between every pair of adjacent gluing patches, and once between the interval $\{0\} \times[0,1]$ and each neighbouring gluing patch. In the case of an annulus which is disjoint from all other annuli we make at least one cut across the annulus in addition to the cut starting at $q_{i}$. The elements of $\mathcal{C}$ shall be called cutting arcs. A curve diagram is said to be reduced if it is in general position with respect to the family $\mathcal{C}$ of cutting arcs and its (geometric) intersection number with any cutting arc is as small as possible within its equivalence class. It is not too hard to see that any diagram is equivalent to a reduced one, and that this reduced representative is unique up to an ambient isotopy through a family of reduced diagrams. We may make this statement more precise as follows.

If we orient each cutting arc transversely, then each diagram $E$ in $F$ which is in general position with respect to $\mathcal{C}$ may be associated with an $n$-tuple $\left(w_{1}, \ldots, w_{n}\right)$ where each $w_{i}$ is a word in the alphabet $\mathcal{C}^{ \pm}=\left\{c, c^{-1}: c \in \mathcal{C}\right\}$ 


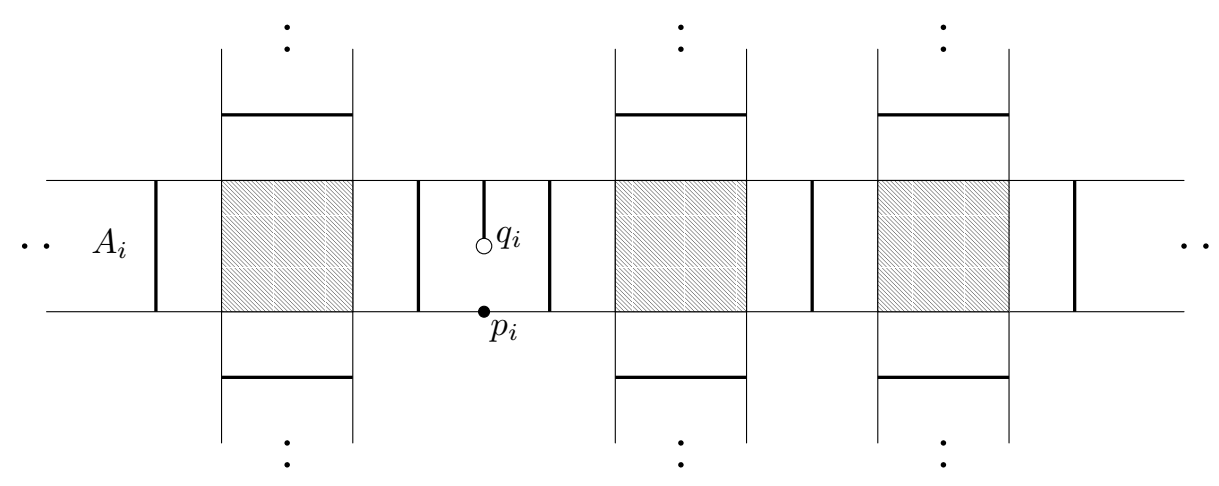

Figure 6: Cutting arcs on the annulus $A_{i}$

obtained by reading off with sign the sequence of crossings that the curve $E_{i}$ makes with $\mathcal{C}$ while travelling from the boundary point $p_{i}$ toward the marked point $q_{i}$. We shall call $\left(w_{1}, \ldots, w_{n}\right)$ the crossing sequence of the diagram $E$, and refer to $w_{i}$ as the crossing sequence of $E_{i}$, for each $i=1, \ldots, n$. A diagram $E$ is reduced if and only if each component of its crossing sequence is a freely reduced word. Moreover, two reduced diagrams which are equivalent have the same crossing sequence. This is simply because any ambient isotopy of diagrams will induce a finite sequence of modifications of the associated crossing sequences which consist only of trivial insertions and deletions.

For each $i=1, \ldots, n$ we define the word $\alpha_{i}$ as follows. Let $E^{(i)}$ denote a reduced diagram equivalent to $\beta_{i}\left(E^{0}\right)$. Then $\alpha_{i}$ is defined as the crossing sequence for $E_{i}^{(i)}$. In other words, $\alpha_{i}$ is the crossing sequence for a reduced path which travels exactly once around $A_{i}$ from $p_{i}$ to $q_{i}$. Let $1 \leq i \leq n$. We say that a reduced diagram $E$ has an $i$-tail if the crossing sequence $w_{i}$ for $E_{i}$ ends with $\alpha_{i}$ or $\alpha_{i}^{-1}$. More generally, we say that any diagram has an $i$-tail if it is equivalent to a reduced diagram with an $i$-tail. By our previous remarks, this notion is well defined on equivalence classes of diagrams in $F$.

Lemma 14 Let $E$ be a diagram in $F$, and let $k \in \mathbb{Z} \backslash\{0\}$.

(i) If $E$ has both an $i$-tail and a $j$-tail, for $i \neq j$, then $\left(a_{i}, a_{j}\right) \in \mathcal{E}$, i.e. the generators $a_{i}$ and $a_{j}$ of $A_{\Delta(S)}$ commute.

(ii) If $E$ does not have an $i$-tail, then $\beta_{i}^{2 k}(E)$ does (have an $i$-tail).

(iii) Let $\left(a_{i}, a_{j}\right) \in \mathcal{E}$. Then $E$ has a $j$-tail if and only if $\beta_{i}^{2 k}(E)$ has a $j$-tail.

Proof Without loss of generality, we may suppose that all diagrams considered are reduced. 
(i) The (reduced) diagram $E$ having an $i$-tail means that the curve $E_{i}$ makes a complete circuit along the length of the annulus $A_{i}$ thus crossing transversely every annulus $A_{k}$ which intersects $A_{i}$ (in fact, crossing every gluing patch). Since the different curves $E_{i}$ and $E_{j}$ of the diagram $E$ are disjoint, it is clear that $E$ cannot therefore have both an $i$-tail and a $j$-tail unless $A_{i}$ and $A_{j}$ are disjoint, in which case $\left(a_{i}, a_{j}\right) \in \mathcal{E}$.

We leave (ii) and (iii) as exercises for the reader. Note that in part (ii) at least a double twist $\beta_{i}^{2}$ is needed to ensure the appearance of an $i$-tail.

To prove Proposition [13, we consider a nontrivial element $x \in A_{\Delta}$. Each nontrivial element can by represented by a nontrivial "reduced expression" as defined below.

An expression for $x \in A_{\Delta}$ of syllable length $\ell$ is a word in the generators $\left\{a_{1}, \ldots, a_{n}\right\}$ representing $x$ and of the form

$$
X=a_{i_{1}}^{k_{1}} \cdots a_{i_{\ell}}^{k_{\ell}} \quad \text { where } k_{j} \in \mathbb{Z} \backslash\{0\} \text { and } i_{j} \in\{1, \ldots, n\} \text { for } 1 \leq j \leq \ell .
$$

Briefly, an amalgamation is simply lumping together adjacent syllables of the same type to shorten the syllable length of $X$, and shuffle equivalence of expressions is generated by exchanges of adjacent syllables $a_{i}^{k}, a_{j}^{m}$ where $\left(a_{i}, a_{j}\right) \in \mathcal{E}$. These operations yield new expressions for the same element of $A_{\Delta}$. A reduced expression is an expression which is not shuffle equivalent to one which admits an amalgamation. The key properties of reduced expressions are that (i) every element $x \in A_{\Delta}$ may be represented by a reduced expression $X$ (obtained from a given expression by a finite process of shuffling and amalgamating), and (ii) any two reduced expressions $X, X^{\prime}$ for the same element $x \in A_{\Delta}$ are shuffle equivalent. We refer to [12] or [14 for more details - see also 7]. Note that property (ii) is a consequence of the following Lemma.

We say that a (reduced) expression ends in $a_{i}$ if it is shuffle equivalent to an expression of the form $a_{i_{1}}^{k_{1}} \cdots a_{i_{\ell}}^{k_{\ell}}$ where $i_{1}=i$.

Lemma 15 Let $X$ be a reduced expression. Then $X$ ends in $a_{i}$ if and only if the diagram $\varphi(X)\left(E^{0}\right)$ has an $i$-tail.

Proof We proceed by induction of $\ell(X)$. Note firstly that, for all $i=1, \ldots, n$, the trivial expression $X=1$ does not end in $a_{i}$ while at the same time $E^{0}=$ $\varphi(1)\left(E^{0}\right)$ does not have an $i$-tail.

Suppose $X$ ends in $a_{i}$, then $X=a_{i}^{k} X^{\prime}$ for some $k \in \mathbb{Z} \backslash\{0\}$ and some reduced expression $X^{\prime}$ where $\ell\left(X^{\prime}\right)=\ell(X)-1$ and $X^{\prime}$ does not end in $a_{i}$. By induction 
$E=\varphi\left(X^{\prime}\right)\left(E^{0}\right)$ has no $i$-tail. Thus, by Lemma14(ii), $\varphi(X)\left(E^{0}\right) \sim \beta_{i}^{2 k}(E)$ does have an $i$-tail, as required.

Now, suppose that $\varphi(X)\left(E^{0}\right)$ has a $j$-tail. We shall show that $X$ ends in $a_{j}$. Since $X$ is necessarily nontrivial there exists an $i$ for which $X$ ends in $a_{i}$, and we may assume that $i \neq j$. By the argument just given we may deduce that $\varphi(X)\left(E^{0}\right)$ has an $i$-tail. But now by Lemma 14(i) we have $\left(a_{i}, a_{j}\right) \in \mathcal{E}$. Writing $X=a_{i}^{k} X^{\prime}$ for some $k \in \mathbb{Z} \backslash\{0\}$ and some reduced expression $X^{\prime}$ with $\ell\left(X^{\prime}\right)=\ell(X)-1$, we have $\varphi(X)\left(E^{0}\right)=\beta_{i}^{2 k}\left(\varphi\left(X^{\prime}\right)\left(E^{0}\right)\right)$ and by Lemma 14(iii) we deduce that $\varphi\left(X^{\prime}\right)\left(E^{0}\right)$ also has a $j$-tail. Now, by induction, $X^{\prime}$ ends in $a_{j}$ and, since the letters $a_{i}$ and $a_{j}$ commute, a single shuffle shows that $X$ also ends in $a_{j}$.

Proof of Proposition 13 Recall that the pure braid group $P B_{n}(S)$ acts on the punctured surface by isotopy classes of homeomorphisms via the homomorphism $\mu: P B_{n}(S) \rightarrow \mathcal{M}(F)$. Let $\varphi^{\prime}$ denote the composition $\mu \circ \varphi$. Our aim is now to prove that $\varphi^{\prime}$ is injective. Note that any nontrivial element $x \in A_{\Delta}$ is represented by a nontrivial reduced expression $X$, which ends in $a_{i}$ say. It follows from Lemma 15 that $\varphi^{\prime}(x)\left(E^{0}\right)$ has an $i$-tail, and hence is not equivalent to the diagram $E^{0}$. That is to say that $\varphi^{\prime}(x)$ is a nontrivial mapping class. Therefore $\varphi^{\prime}$ is injective.

Remark 16 Note that, in order to show that the group $A_{\Delta}$ embeds in the pure $n$-string braid group of $S$ we have constructed a faithful representation $\varphi^{\prime}: A_{\Delta} \rightarrow \mathcal{M}(F)$ of $A_{\Delta}$ in the mapping class group of the $n$-punctured surface $S$ such that $\varphi^{\prime}$ factors through $P B_{n}(S)$. In fact it is not too hard to see that the image of $\varphi^{\prime}$ is actually realizable in the sense of Nielsen and Kerckhoff 17]: $\operatorname{im}\left(\varphi^{\prime}\right)$ can be realised by a group of diffeomorphisms of $S$ which fix $\partial S$ and the distinguished points $q_{1}, \ldots, q_{n}$. In other words $\varphi^{\prime}$ factors through an (injective) homomorphism $f: A_{\Delta} \rightarrow \operatorname{Diffeo}\left(S ; \partial S, q_{1} \ldots q_{n}\right)$. This is because, for each $i=1,2, \ldots, n$, the mapping class $\varphi^{\prime}\left(a_{i}\right)=\mu\left(\beta_{i}^{2}\right)$ may be represented by a diffeomorphism $\alpha_{i}$ which is the identity outside the annulus $A_{i}$. In the case that $\left(a_{i}, a_{j}\right) \in \mathcal{E}$, the annuli $A_{i}$ and $A_{j}$ are disjoint subsets of $S$ and it is clear that $\alpha_{i}$ and $\alpha_{j}$ commute as diffeomorphisms. Thus all of the relations in the standard presentation of $A_{\Delta}$ are satisfied in the group $\operatorname{Diffeo}\left(S ; \partial S, q_{1} \ldots q_{n}\right)$.

\section{Embeddings in the classical braid groups}

So far we have developed a method for embedding right-angled Artin groups in surface braid groups. It seems a much harder task to find embeddings in 
classical braid groups. It is nevertheless conceivable that this might be possible for all right-angled Artin groups, and in this section we present some partial results in this direction.

Given a finite simple graph $\Delta$, we define the dual or opposite graph $\Delta^{\mathrm{op}}$ to be the finite simple graph with the same vertex set as $\Delta$ where $(u, v)$ is defined to be an edge of $\Delta^{\mathrm{op}}$ precisely when $u$ and $v$ are distinct non-adjacent vertices of $\Delta$.

Proposition 17 Let $A_{\Delta}$ be the right-angled Artin group defined by the graph $\Delta$. If the opposite graph $\Delta^{\mathrm{op}}$ is a planar graph then the $A_{\Delta}$ embeds as a subgroup of the classical pure braid group $P B_{l}$ for sufficiently large $l \in \mathbb{N}$, and also in $\operatorname{Diff}\left(D^{2} ; \partial D^{2}, p_{1} \ldots p_{l}\right)$.
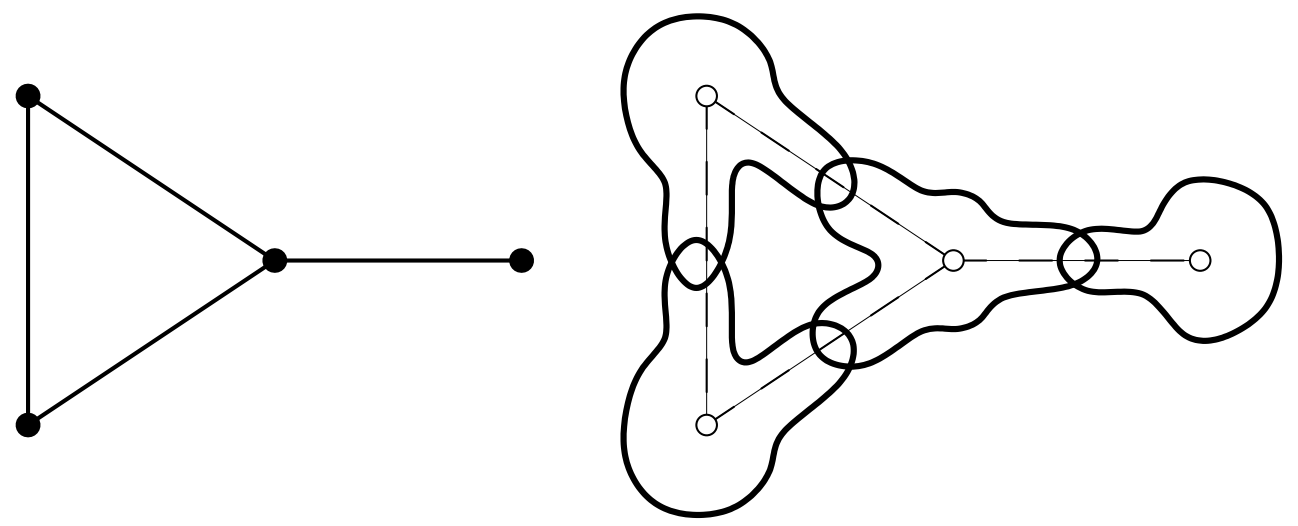

Figure 7: How to construct a circle diagram for a planar surface $S$ such that $\Delta(S)=\Delta$, starting from the planar dual graph $\Delta^{\text {op }}$ (shown on the left).

Proof One can use the construction method suggested in Figure 7 to obtain a planar surface $S$ such that $\Delta(S)=\Delta$. By Theorem 10 we obtain an embedding of $A_{\Delta}$ in the pure braid group, and the diffeomorphism group, of $S$. By glueing a twice-punctured disk to all but one of the components of $\partial S$ and extending all automorphisms of $S$ by the identity on these twice-punctured disks, we get faithful representations in the the braid groups and the diffeomorphism groups, respectively, of an $l$-punctured disk (see Theorem 2.3 of [22], also [23]).

Example 18 Let $\Delta$ be the hexagonal graph shown in Figure 8 (b). The rightangled Artin group $A_{\Delta}$ contains the fundamental group of the orientable surface 

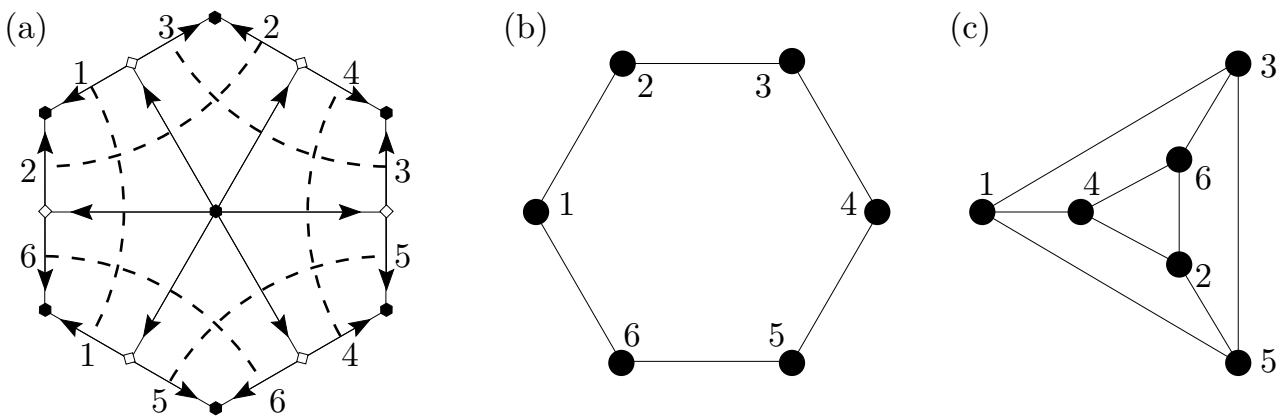

Figure 8: (a) A cubing of the orientable surface of genus 2, with the six midplanes marked in dashed lines. The surface is obtained by identifying edges with the same label. (b) The graph $\Delta$ symbolizing the right-angled Artin group in which $\pi_{1}(S)$ can be embedded. (c) The opposite graph $\Delta^{\text {op }}$ is planar.

of genus 2 (see Figure 8(a)) which is embedded by the technique of Section 4 The opposite graph $\Delta^{\mathrm{op}}$ (shown in Figure 8(c)) is planar and therefore, by Proposition 17 the group $A_{\Delta}$ (together with the surface subgroup) embeds in a classical pure braid group $P B_{l}(l=44$ suffices $)$.

Proposition 19 If the graph $\Delta^{\mathrm{op}}$ has a finite-sheeted covering which is planar, then the right-angled Artin group symbolised by $\Delta$ embeds in the classical pure braid group $P B_{l}$ for sufficiently large $l$, and also in $\operatorname{Diff}\left(D^{2} ; \partial D^{2}, p_{1} \ldots p_{l}\right)$.

Proof Let $\widetilde{\Delta}^{\text {op }}$ be the planar cover of $\Delta^{\text {op }}$ (with say $k$ sheets). We define $\widetilde{A}_{\Delta}$ to be the right-angled Artin group whose defining graph is the opposite graph of $\widetilde{\Delta}^{\mathrm{op}}$. There is an obvious homomorphism $j: A_{\Delta} \rightarrow \widetilde{A}_{\Delta}$, given by sending a generator $h$ of $A_{\Delta}$ to the $k$-letter word $\widetilde{h}^{(1)} \ldots \widetilde{h}^{(k)}$, where $\widetilde{h}^{(1)}, \ldots, \widetilde{h}^{(k)}$ are the generators of $\widetilde{A}_{\Delta}$ covering $h$. Note that $\widetilde{h}^{(1)}, \ldots, \widetilde{h}^{(k)}$ commute in $\widetilde{A}_{\Delta}$.

We claim that the homomorphism $j: A_{\Delta} \rightarrow \widetilde{A}_{\Delta}$ is injective. To see this, we consider a word $w$ in the letters $h_{1}^{ \pm 1}, \ldots, h_{m}^{ \pm 1}$ which is a representative of the smallest possible length of $[w] \in A_{\Delta}$. Equivalently, by Proposition 9 , $w$ is $\Delta$-reduced: it has no subword of the form $h_{i} w^{\prime} h_{i}^{-1}$ (or $h_{i}^{-1} w^{\prime} h_{i}$ ), where $i \in\{1, \ldots, m\}$, and $w^{\prime}$ is a word consisting entirely of letters that commute with $h_{i}$. But then the "obvious" representative of $j([w])$ (given by the letter-by-letter substitution $\left.h_{i} \mapsto \widetilde{h}_{i}^{(1)} \ldots \widetilde{h}_{i}^{(k)}\right)$ satisfies the same condition. It follows that this word is also of the smallest possible length, and in particular $j([w]) \neq 0$. (See 7] for a similar argument in a slightly different situation).

Thus $A_{\Delta}$ embeds in $\widetilde{A}_{\Delta}$, which in turn embeds in $B_{l}$ and $\operatorname{Diff}\left(D^{2} ; \partial D^{2}, p_{1} \ldots p_{l}\right)$ for sufficiently large $l$, by Proposition 17. 
Example 20 The graph $\Delta\left(K_{3,3}\right)$ shown in Figure 1 is self-dual. That is, $\Delta\left(K_{3,3}\right)^{\text {op }} \cong \Delta\left(K_{3,3}\right)$. This graph is not planar, but it has a planar double cover (since it embeds in a Möbius band). This implies that the fundamental group of the nonorientable surface of Euler characteristic -3 embeds in a pure classical braid group, using Proposition 19.

There are however many examples of right-angled Artin groups (notably the groups $\mathbb{Z}^{n}$ ) which embed in $P B_{l}$, for some $l$, but for which the above propositions either do not apply, or do not provide the best strategy. It might be that in many individual cases the direct approach of drawing circles in the plane, such as is illustrated in Figure 5] (see Remark 12), is the most efficient. These drawings yield embeddings in planar surface braid groups and as explained in the proof of Proposition 17 the planar surfaces may be embedded in multipunctured disks in order to obtain embeddings in $P B_{l}$ for a suitable $l$.
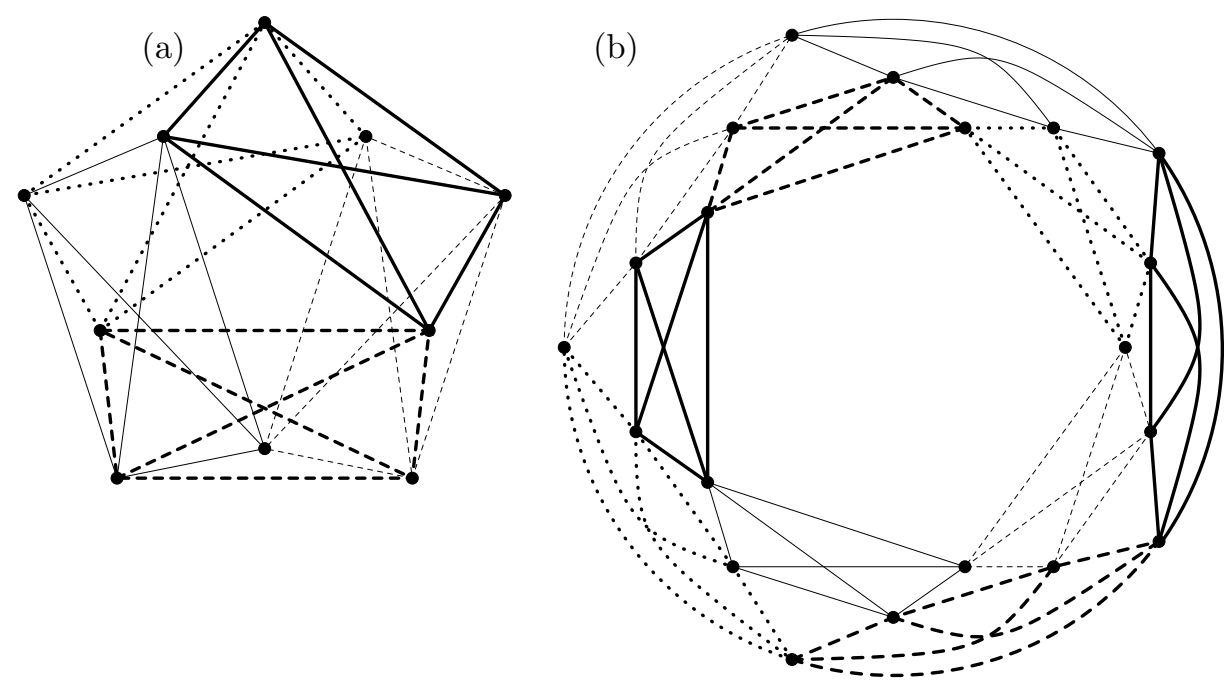

Figure 9: (a) The graph $\Delta\left(K_{5}\right)^{\mathrm{op}}$; and (b) a double cover $\widetilde{\Delta}^{\mathrm{op}}$ of the graph in (a).

Example 21 Consider $\Delta=\Delta\left(K_{5}\right)$. Recall that $A_{\Delta\left(K_{5}\right)}$ contains an embedded copy of the graph braid group $R B_{2}\left(K_{5}\right)$ which is isomorphic to the fundamental group of the closed surface of Euler characteristic -5 . The circle diagram of Figure 5(c) yields an embedding of $A_{\Delta}$ in a planar surface braid group, and hence in a classical pure braid group. This example may alternatively be treated by a combination of ideas, as follows. The graph $\Delta^{\mathrm{op}}$ is shown in Figure 9(a). Passing to the double cover $\widetilde{\Delta}^{\text {op }}$ shown in Figure 9(b), it suffices by the argument of Proposition [19] to consider the right-angled Artin 
group whose defining graph is dual to $\widetilde{\Delta}^{\text {op }}$. It is now a relatively easy matter to produce an appropriate circle diagram by modifying the idea of Figure 7 as suggested by Figure 10 in order to embed the latter group in a classical pure braid group.

Note that this, and every embedding discussed in this section, factors through a group of actual diffeomorphisms of the $l$ times punctured disk, as explained in Remark [16] of the previous section.
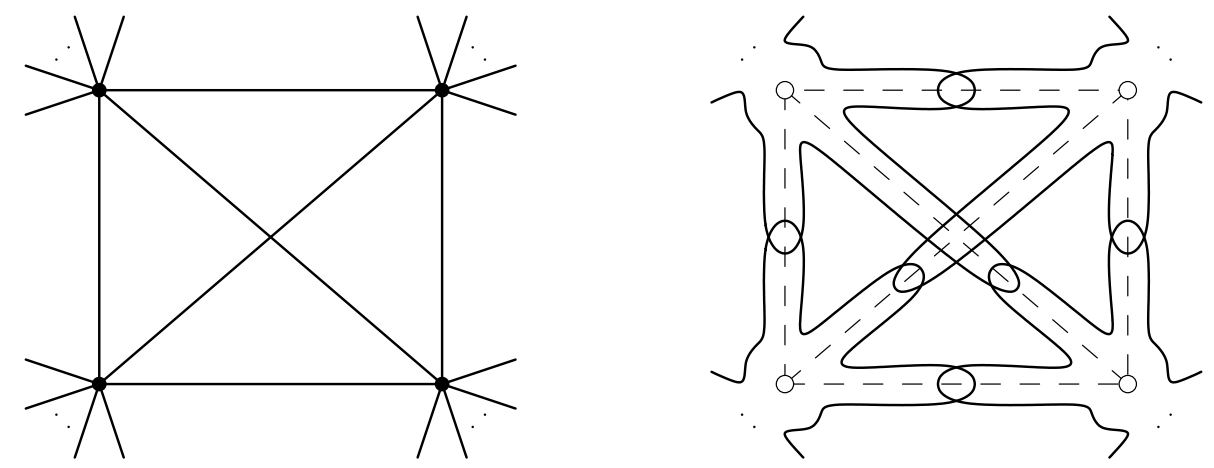

Figure 10: How to modify the construction of the circle diagram for the surface $S$ at each of the self-intersection points in the planar projection of the graph $\widetilde{\Delta^{\mathrm{op}}}$.

The circle diagrams in Figure 5 allow one to realise embeddings in a certain $P B_{l}$ for each of the right-angled Artin groups used in Section 4. As a consequence, we have the following:

Proposition 22 With the exception of $R P^{2}, K l$ and $S_{-1}$, the fundamental group of every surface embeds in a pure braid group $P B_{l}$ for some $l$.

Observe that the group $\pi_{1}\left(R P^{2}\right)$ of order 2 never embeds (since the braid groups are torsion free), and $\pi_{1}(K l)$ cannot embed in a pure braid group since the latter are bi-orderable while $\pi_{1}(K l)$ is not (it has generalised torsion). However, we do not know whether $\pi_{1}(K l)$ embeds in $B_{l}$ for some $l$, or whether $\pi_{1}\left(S_{-1}\right)$ embeds in either $B_{l}$ or $P B_{l}$ for some $l$.

Finally, we make the following observation:

Proposition 23 Let $G$ denote a finite graph. If $G$ or some finite index cover of $G$ is a planar graph, then, for any $n \geq 2$, the reduced braid group $R B_{n}(G)$ embeds in a pure braid group $P B_{l}$ for some $l$. 
Proof If $G$ itself is planar, then $\Delta(G)^{\text {op }}$ is also planar and may be constructed (in the plane) as follows: place a vertex of $\Delta(G)^{\mathrm{op}}$ at the midpoint of each edge of $G$, and an edge between two such vertices if the corresponding edges of $G$ share a common vertex in $G$. Note also that, if $G \rightarrow G_{0}$ is a finite index covering map (with $G$ planar), then there is a naturally induced covering map $\Delta(G)^{\text {op }} \rightarrow \Delta\left(G_{0}\right)^{\text {op }}$. The result now follows by Proposition 19 ,

We do not know whether every reduced braid group may be embedded in a pure braid group $P B_{l}$. In connection with Propositions [19] and 23] we note that it seems to be an open question (or conjecture) as to whether every graph which has a planar finite cover has in fact a planar double cover (or is already planar), attributed to Henry Glover - see Bestvina's "Questions in Geometric Group Theory" problem list.

Note, finally, that since $K_{3,3}$ and $K_{5}$ both embed in a Möbius band and therefore have planar double covers, Proposition 23 gives yet another method for embedding the closed Euler characteristic -3 and -5 surface groups discussed above.

\section{References}

[1] A Abrams, Configuration Spaces and Braid Groups of Graphs, PhD thesis, University of California at Berkeley (2000)

[2] A Abrams, Configuration spaces of colored graphs, Geometriae Dedicata, to appear

[3] A Abrams, R Ghrist, Finding topology in a factory: configuration spaces, Amer. Math. Monthly 109 no. 2 (2002) 140-150

[4] M Bestvina, N Brady, Morse theory and finiteness properties of groups, Invent. Math. 129 (1997), no. 3, 445-470

[5] M R Bridson, A Haefliger, Metric Spaces of Non-Positive Curvature, Springer-Verlag (1999)

[6] R Charney, The Tits conjecture for locally reducible Artin groups, Internat. J. Algebra Comput. 10 no. 6 (2000) 783-797

[7] J Crisp, L Paris, The solution to a conjecture of Tits on the subgroup generated by the squares of the generators of an Artin group, Invent. Math. 145 (2001) 1936

[8] C Droms, B Servatius, H Servatius, Surface subgroups of graph groups, Proc. Amer. Math. Soc. 106 (1989), no. 3, 573-578

[9] G Duchamp, J-Y Thibon, Simple orderings for free partially commutative groups, Internat. J. Algebra Comput. 2 (1992), no. 3, 351-355 
[10] J Gonzalez-Meneses, Ordering pure braid groups on compact, connected surfaces, Pacific J. Math. 203 (2002), 369-378

[11] C Gordon, D D Long, A W Reid, Surface subgroups of Coxeter and Artin groups. J. Pure Appl. Algebra 189 (2004), no. 1-3, 135-148.

[12] E R Green, Graph Products of Groups, Thesis, The University of Leeds, 1990.

[13] M Gromov, Hyperbolic Groups, Essays in Group Theory (S M Gersten, ed), Springer-Verlag, MSRI Publ. 8 (1987) 75-263

[14] S Hermiller, J Meier, Algorithms and geometry for graph products of groups, J. Algebra 171 (1995), no. 1, 230-257

[15] T Hsu, D T Wise, On linear and residual properties of graph products, Michigan Math. J., to appear.

[16] S P Humphries, On representations of Artin groups and the Tits conjecture, J. Algebra 169 (1994), no. 3, 847-862

[17] S Kerckhoff, The Nielsen realisation problem, Ann. of Math. (2) 117 (1983) 235-265

[18] R C Lyndon, The equation $a^{2} b^{2}=c^{2}$ in free groups, Michigan Math. J. 6 (1959) 155-164

[19] R C Lyndon, P E Schupp, Combinatorial Group Theory, Springer-Verlag, Berlin (1977).

[20] R C Lyndon, MP Schützenberger, The equation $a^{M}=b^{N} c^{P}$ in a free group, Michigan Math. J. 9 (1962) 289-298

[21] G Niblo, L Reeves, The geometry of cube complexes and the complexity of their fundamental groups, Topology 37 (1998) 621-633

[22] L Paris, D Rolfsen, Geometric subgroups of surface braid groups, Ann. Inst. Fourier 49 (1999),101-156.

[23] L Paris, D Rolfsen, Geometric subgroups of mapping class groups, J. Reine Angew. Math. 521 (2000),47-83.

[24] D Rolfsen, B Wiest, Free group automorphisms, invariant orderings and topological applications, Algebr. Geom. Topol. 1 (2001) 311-320

[25] H Servatius, Automorphisms of graph groups, J. Algebra 126 (1989) 34-60

[26] L VanWyk, Graph groups are biautomatic, J. Pure Appl. Algebra 94 (1994), no. $3,341-352$

[27] C Wrathall, Free partially commutative groups, Combinatorics, computing and complexity (Tianjing and Beijing, 1988), 195-216, Math. Appl. (Chinese Ser.), 1, Kluwer Acad. Publ., Dordrecht, 1989

Institut de Mathématiques de Bourgogne (IMB), UMR 5584 du CNRS

Université de Bourgogne, 9 avenue Alain Savary, B.P. 47870

21078 Dijon cedex, France

IRMAR, UMR 6625 du CNRS, Campus de Beaulieu, Université de Rennes 1 35042 Rennes, France

Email: jcrisp@u-bourgogne.fr, bertold.wiest@math.univ-rennes1.fr

Received: 10 April 2003 